\title{
Investigation into the underlying regulatory mechanisms shaping inflorescence architecture in Chenopodium quinoa
}

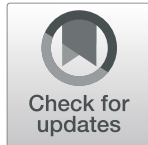

Qi Wu ${ }^{1,2^{* \dagger}} \mathbb{B}$, Xue Bai ${ }^{1,2+}$, Wei Zhao ${ }^{1,2+}$, Xiaodong Shi ${ }^{1,2}$, Dabing Xiang ${ }^{1,2}$, Yan Wan ${ }^{1,2}$, Xiaoyong Wu ${ }^{1,2}$, Yanxia Sun ${ }^{1,2}$, Jianglin Zhao ${ }^{1,2}$, Lianxin Peng ${ }^{1,2}$ and Gang Zhao ${ }^{1,2}$

\begin{abstract}
Background: Inflorescence architecture is denoted by the spatial arrangement of various lateral branches and florets formed on them, which is shaped by a complex of regulators. Unveiling of the regulatory mechanisms underlying inflorescence architecture is pivotal for improving crop yield potential. Quinoa (Chenopodium quinoa Willd), a pseudo cereal originated from Andean region of South America, has been widely recognized as a functional super food due to its excellent nutritional elements. Increasing worldwide consumption of this crop urgently calls for its yield improvement. However, dissection of the regulatory networks underlying quinoa inflorescence patterning is lacking.
\end{abstract}

Results: In this study, we performed RNA-seq analysis on quinoa inflorescence samples collected from six developmental stages, yielding a total of 138.8 GB data. We screened 21,610 differentially expressed genes (DEGs) among all the stages through comparative analysis. Weighted Gene Co-Expression Network Analysis (WGCNA) was performed to categorize the DEGs into ten different modules. Subsequently, we placed emphasis on investigating the modules associated with none branched and branched inflorescence samples. We manually refined the coexpression networks with stringent edge weight cutoffs, and generated core networks using transcription factors and key inflorescence architecture related genes as seed nodes. The core networks were visualized and analyzed by Cytoscape to obtain hub genes in each network. Our finding indicates that the specific occurrence of B3, TALE, WOX, LSH, LFY, GRAS, bHLH, EIL, DOF, G2-like and YABBY family members in early reproductive stage modules, and of TFL, ERF, bZIP, HD-ZIP, C2H2, LBD, NAC, C3H, Nin-like and FAR1 family members in late reproductive stage modules, as well as the several different MADS subfamily members identified in both stages may account for shaping quinoa inflorescence architecture.

Conclusion: In this study we carried out comparative transcriptome analysis of six different stages quinoa inflorescences, and using WGCNA we obtained the most highly potential central hubs for shaping inflorescence. The data obtained from this study will enhance our understanding of the gene network regulating quinoa inflorescence architecture, as well will supply with valuable genetic resources for high-yield elite breeding in the future.

Keywords: Chenopodium quinoa, Inflorescence architecture, RNA-seq, Transcriptome analysis, Weighted gene coexpression network analysis

\footnotetext{
*Correspondence: jerviswuqi@126.com

${ }^{\dagger}$ Qi Wu, Xue Bai and Wei Zhao contributed equally to this work.

'Key Laboratory of Coarse Cereal Processing Ministry of Agriculture and Rural

Affairs, College of Pharmacy and Biological Engineering, Chengdu University,

Chengluo road 2025, Shiling town, Longquanyi District, Chengdu 610106,

Sichuan province, People's Republic of China

${ }^{2}$ National Research and Development Center for Coarse Cereal Processing,

College of Pharmacy and Biological Engineering, Chengdu University,

Chengdu 610106, People's Republic of China
}

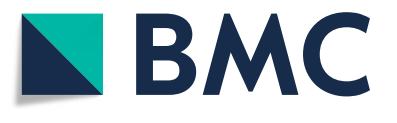

(c) The Author(s). 2019 Open Access This article is distributed under the terms of the Creative Commons Attribution 4.0 International License (http://creativecommons.org/licenses/by/4.0/), which permits unrestricted use, distribution, and reproduction in any medium, provided you give appropriate credit to the original author(s) and the source, provide a link to the Creative Commons license, and indicate if changes were made. The Creative Commons Public Domain Dedication waiver (http://creativecommons.org/publicdomain/zero/1.0/) applies to the data made available in this article, unless otherwise stated. 


\section{Background}

Crop yield is mainly determined by three major factors: grain number per panicle, effective panicle number, and seed setting rate [1]. Under limited growth area increasing the grain number per panicle or individual plant is a fundamental and proficient way to improve the yield. This goal could be achieved via improving the inflorescence architecture. Inflorescence architecture refers to the space arrangement of florets and branches that ultimately form the grains. It is shaped by a complex of post-embryogenesis developmental processes [2-5]. Variation of inflorescence architecture occurs among different species [4]. In Arabidopsis thaliana after transition from vegetative phase to reproductive phase, a shoot apical meristem (SAM) converts into a main inflorescence meristem (IM). Subsequently, the IMs bear axillary meristems (AMs) that sequentially form floral meristems (FMs) or indeterminate branch meristems (BMs) $[4,6,7]$. The growth of an IM will stay indefinite before senescence. By contrast, in grass species such as rice (Oryza sativa), maize (Zea mays) and oat (Avena sativa), the AMs on the main IM produce primary (pBMs), secondary (sBMs) or highorder branch meristems on which spikelet meristems (SMs) and FMs are ultimately formed [6, 7]. The growth of grass IM is definite and terminated by flowers. Hence, where and when the BMs and FMs/SMs are formed along an IM, in other word, the identities and activities of various meristems can directly influence the inflorescence morphology $[4,5]$. Over past few decades, combination of plant morphology, genetics and molecular biology approaches has unveiled many genes involved in those events, which significantly contributes to our understanding of the inflorescence architecture regulatory networks.

The classical CLAVATA (CLV)-WUSCHEL (WUS) feed-back regulatory loop is indispensable for maintaining the activity of pluripotent stem cells in the central region of SAM, which guarantees the potential to continuously generate leaf primordia or reproductive organs $[4,6,7]$. clv1, clv2 and clv3 mutants all display enlarged inflorescence size and increased floral organs due to overproliferation of stem cells [8-10]. Similarly, many evidences showed that mutations in rice CLV1 homolog FLORAL ORGAN NUMBER 1 (FON1) [11], CLV3 homolog FON4/2 [12], and in maize CLV1 homolog THICK TASSEL DWARF 1 (TD1) [13], CLV2 homolog FASCIATED EAR 2 (FEA2) [14] led to increased floral organ number and inflorescence size. The homeodomain-containing transcription factor (TF) WUS promotes the expression of CLV3 which encodes a small CLV3/ESR-related (CLE) peptide $[15,16]$. In turn $C L V 3$ represses the expression WUS. Another homeodomain TF-Class 1 KNOTTED 1-like homeobox (KNOX), which sophisticatedly controls the homeostasis between gibberellin and cytokinin, is also essential for establishment and maintainence of SAM [17].
The corresponding KNOX mutants in rice (Oryza sativa HOMEOBOX 1, OSH1) [18], maize (KNOTTED 1, KN1) [19], and Arabidopsis (SHOOT MERISTEMLESS, STM) [20] are defective in inflorescence morphology.

At optimal time the florigen proteins move from leaves to SAM to trigger downstream flowering programs, thus acquiring IM identity [3]. This process is very important for panicle structure and the ultimate grain yield in grass crops. Several rice MADS-box genes including OsMADS14, OsMADS15, OsMADS18, and OsMADS34 (also called PANICLE PHYTOMER 2, PAP2) are demonstrated to play crucial roles in IM specification [21, 22]. Interestingly, their Arabidopsis counterparts such as APETALA 1 (AP1) and SEPALLATA $(S E P)$-like MADS-box genes are responsible for floral organ identity determination [23-25], indicating divergent roles of those homologs between different species. The number of BMs produced on the IMs determines the final inflorescence branches [5]. The phosphatidylethanolamine-binding protein (PEBP) encoding gene TERMINAL FLOWER 1 (TFL1) antagonizes with $A P 1$ and $L E A F Y$ (LFY) to coordinately modify inflorescence branching [23]. Overexpression of the rice TFL1 homologs REDUCED CULM NUMBER 1 (RCN1) and RCN2 [26], or Zea mays homologs CENTRORADIALIS 1 (ZCN1) to ZCN6 [27] delays the conversion from IMs or BMs to FMs, thereby generating highly branched inflorescences. Consistently, the Arabidopsis tfl1 mutant generates a determinate inflorescence [28]. Conversely, LFY, a plant-specific $\mathrm{TF}$, acts upstream of and promotes the expression of AP1 to specify FMs determination via suppression of TFL1 [23]. Loss of function of $L F Y$ homologs results in hampered floral identify acquisition in Arabidopsis and Petunia [29]. Besides, some other MADS-box genes, including SHORT VEGETATIVE PHASE (SVP), SUPPRESSOR OF OVEREXPRESSION OF CONSTANS 1 (SOC1), AGAMOUS-LIKE 24 (AGL24) and SEP4, interact with $A P 1$ and function redundantly to repress the expression of TFL1 [30]. Knockdown of rice SVP/AGL24 homologs (OsMADS22, OsMADS47, and OsMADS55) and SOC1 homologs (OsMADS50, OsMADS56) in OsMADS34 (homolog of SEP4) mutant background leads to significantly enhanced branching panicle which mimics the phenotypes of $R C N$ overexpressors [30]. OsSPL14, a SQUAMOSA promoter binding protein-like (SBP) box TF responsible for a rice yield quantitative trait loci (QTL) Ideal Plant Architecture 1 (IPA1)/Wealthy Farmer's Panicle (WFP), positively regulates the primary branch number and yield [31]. The maize SBP TF-TASSELSHEATH4 (TSH4) acts downstream of meristem identity genes including RAMOSA1 (RA1), RA2, RA3 and BRANCHED SILKLESS1 (BD1) to modify the growth of lateral branches [32].

The number of SMs/FMs formed on IMs largely influences the final grain yield [5], and this developmental 
process is regulated by a series of key genes. The rice UNUSUAL FLORAL ORGAN (UFO) homolog ABERRANT PANICLE ORGANIZATION 1 (APO1) depresses transition from IMs to SMs [33]. Similarly, the interacting protein of APO1, APO2/RICE FLORICAULA (RFL) /OsLFY, acts as a suppressor for the transition from IMs to FMs in rice, and the corresponding mutants harbor decreased branched panicle [34]. Remarkably, the functions of rice $A P O 1$ and $A P O 2 / R F L$ are distinguished from their Arabidopsis counterparts which are positive determinants for floral organ specification. Further investigation indicates the divergent roles of $A P O 1$ and $A P O 2 / R F L$ homologs between different species may arise from the distinct expression patterns and functional modes of $L F Y$ genes. Recently, accumulating studies indicate that the Arabidopsis LSH1 and Oryza G1 (ALOG) family proteins are vital modulators of inflorescence structure. TAWAWA1 (TWA1) is a rice nuclear-localized ALOG protein [35]. It prolongs the indeterminate status of IMs to limit the formation of SMs by inducing the expression of SVP-like genes (such as OsMADS22, OsMADS47, and OsMADS55) while depressing the floral organ formation related genes (such as OsMADS7, OsMADS3, and OsMADS58) [35]. Up-regulation of TWA1 gives rise to highly-branched panicle whereas down-regulation of TWA1 leads to an opposite phenotype [35]. The tomato (Solanum lycopersicum) TWA1 homolog TERMINATING FLOWER (TMF) functions in a similar manner as does in rice, and tmf mutants produce single flower-terminated primary branches rather than sympodial inflorescences [36]. Furthermore, Transition from SMs to FMs is also closely associated with the final panicle structure [5]. Evidences obtained from multiple grass species indicate that ethylene-response factor (ERF)-class AP2 TFs act as pivotal mediators for this process. For instance, rice FRIZZY PANICLE (FZP)/BRANCHED FLORETLESS 1 (BFL1) [37], SUPERNUMERARY BRACT (SNB) [38] and MULTI-FLORET SPIKELET 1 (MFS1) [39], maize BD1 and INDETERMINATE SPIKELET 1 (IDS1) [40], and Brachypodium distachyon MORE SPIKELETS 1 (MOS1, [41]) are all essential for triggering FMs specification.

The knowledge acquired from model plants provides significant insights into the inflorescence formation and could be appropriately adopted for investigating and improving the crops yield. Quinoa (Chenopodium quinoa) is an ancient pseudocereal of Amaranthaceae family originating from Andean region in South America [42]. It has been widely recognized as an extraordinary "superfood" because of its abundant and excellent-balanced nutritional components [43], which is especially highlighted by declaration of 2013 as the International Year of Quinoa by United Nations [44]. From the beginning of domestication and cultivation of quinoa in about 5000 7000 years ago, to date growing of quinoa has expanded to more than 100 countries [45]. However, the worldwide rapid increasing consumption of quinoa urgently demands more breeding efforts to improve the yield-related agronomic traits $[44,45]$. Deciphering and understanding of the molecular mechanisms shaping quinoa inflorescence architecture is necessary and helpful to fulfill this purpose. Previously, a few studies have well detailed the sequential changes in quinoa inflorescence morphology [46]. There are two major types of inflorescence architecture in quinoa: Type I: Glomerulate inflorescence; Type II: amaranthiform inflorescence. After floral transition, the inflorescence arises from the apical of the plant or from leaf axils along the stem. As the inflorescence elongates, the secondary branches are formed along with the main axis. As growth goes on, a group of florets may form directly on the secondary branches (Type II), or form on the tertiary branches which are born on the secondary branches (Type I). Thus, Type II inflorescence lacks tertiary branches compared with Type I. The timing of branching (secondary and tertiary) and formation of florets determine the ultimate inflorescence structure. The preliminary studies have enhanced our understanding of the quinoa inflorescence morphogenesis, however, investigation regarding to the in-depth regulatory networks is lacking. Due to the unavailability of quinoa genome sequences before 2017, dissecting the genetic basis by quantitative trait locus (QTL) mapping is limited. Compared with the many evidences related to inflorescence patterning found in model plants such as Arabidopsis and rice, to our knowledge, up to date no genes have been discovered to participate in quinoa inflorescence patterning.

The advances of high-throughput next generation sequencing (NGS) technology in analyzing global transcription regulation combined with the recently released high-quality quinoa reference genome provides us a good opportunity to explore the complexity of transcription regulation shaping inflorescence. In the present study, we performed the first attempt to decipher the molecular basis of quinoa inflorescence regulation. We carried out comparative transcriptome analysis of quinoa inflorescence samples collected from six different developmental stages, and then we generated core transcriptional regulatory networks using Weighted Gene Co-Expression Network Analysis (WGCNA) method and identified several sets of potential candidates regulating quinoa inflorescence structure. The data obtained from this study is very helpful to enhance our understanding of the gene network regulating quinoa inflorescence architecture, and also it will provide highly potential candidates which could be manipulated for elite cultivars breeding in the future. 


\section{Results}

RNA sequencing of different-stage quinoa inflorescences tissues

The quinoa variety "LL-1" plants used in this study were grown in a greenhouse in Chengdu University. "LL-1" is a Type I inflorescence quinoa. The inflorescence samples were collected on December 15th, 2017 from three to five quinoa plants which were sown on July 19th, July 29th, August 24th, September 2nd, September 9th and September 16th, 2017, respectively referring to as 149 days after sowing (DAS), 139 DAS, 113 DAS, 104 DAS, 97 DAS and 90 DAS (Fig. 1). Sampling was performed with three biological replicates for each time point. YP1 (young panicle), YP2, YP3, YP4, P1 (elder panicle) and P2 (Fig. 1, a-f) were used to denote for the materials ranging from early reproductive to late reproductive stages. At phase YP1 (Fig. 1, a), the floral bud did not penetrate through the bracts, but actually the small floral bud was visible when manually removing the surrounding bracts. At YP1 the plants had just finished floral transition, apical meristem was changed to young inflorescence, the branches have not been formed (Fig. 1, a). At YP2, the main inflorescence began to elongate, and the secondary branches began to form (Fig. 1, b). At YP3, the lateral secondary branches were shortly elongated (Fig. 1, c). At YP4, the lateral secondary branches were more elongated and the tertiary branches began to form (Fig. 1, d). At P1, the secondary and tertiary branches were largely elongated and the florets on the lateral branches were formed (Fig. 1, e). At P2, the branches continued elongating, more secondary and tertiary branches were formed and the inflorescence was nearly coming to anthesis stage (Fig. 1, f).
Eighteen libraries were sequenced using Illumina Hiseq2500 platform, yielding an average of about 26 million of paired-end reads $(2 \times 150 \mathrm{bp})$ per library, with the total size amounting to about $138.8 \mathrm{~Gb}$. The Q20 and Q30 values of each library were larger than 97 and 93\% (Table 1), respectively. Subsequently, the clean reads were mapped to the quinoa genomic sequences [42] (NCBI, Chenopodium quinoa, ASM168347v1) using HISAT [47] program. Approximately $96 \%$ of the clean reads were mapped to the reference genome, with $91 \%$ mapped to exon regions and about 4-6\% mapped to intergenic regions (Table 1). Meanwhile, the transcripts identified by StringTie based network flow algorithm [48] without annotation on the reference genome were designated as novel genes (Additional file 13: Dataset 2). The relative expression level of each gene was normalized with Fragments Per Kilobase of transcript sequence per Millions base pairs sequenced (FPKM) method [49]. Pearson correlation coefficient between three biological replicates of different stage tissues varied from 0.916 to 0.988, indicating the replicates were highly similar (Additional file 1: Figure S1). We defined a gene with the $F P K M \geq 1$ in at least one stage as an expressed one. Based on such criteria, we detected 23,951, 24,426, 24, 794, 24,786 expressed genes in YP1, YP2, YP3 and YP4, respectively, while a higher number of expressed genes appeared in P1 $(26,770)$ and P2 $(25,592)$ (Additional file 3: Table S1). Among the expressed genes, 20,748 genes displayed common expression patterns with their FPKM $\geq 1$ at all the stages (Fig. 2). Then, we analyzed the transcriptome data to find out the genes conferred FPKM $\geq 1$ exclusively at one stage but FPKM $<1$ at the other stages. Consequently, we found that 358, 151, 232 and 152
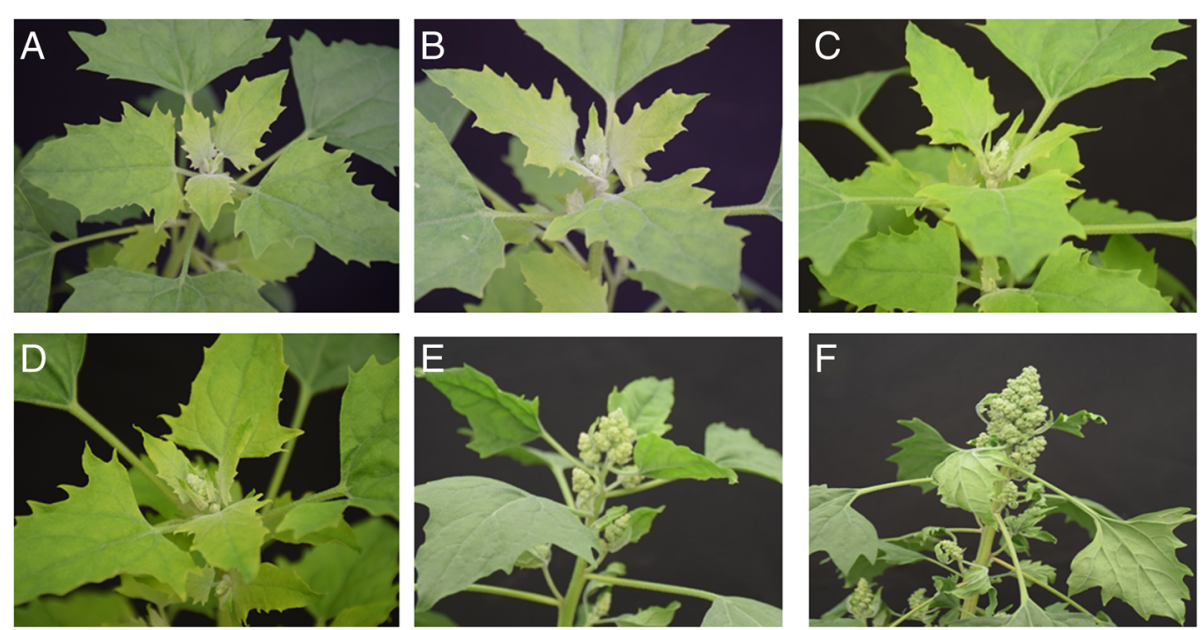

Fig. 1 The morphologies of quinoa inflorescence at different developmental stages. The quinoa seeds were sown on September 16 th (a), September 9th (b), September 2nd (c), August 24th (d), July 29th (e), and July 19th (f), 2017. The quinoa plants were grown under nature photoperiod with regular management in a greenhouse at Chengdu University. The inflorescence samples were collected on December 15 th, 2017 and were denoted as YP1 (a), YP2 (b), YP3 (c), YP4 (d), P1 (e) and P2 (f) for RNA sequencing 
Table 1 Summary of the RNA-seq data in this study yielded from Illumina Hiseq2500 platform

\begin{tabular}{|c|c|c|c|c|c|c|c|c|c|c|}
\hline Sample & $\begin{array}{l}\text { Raw reads } \\
\text { (nt) }\end{array}$ & $\begin{array}{l}\text { Clean } \\
\text { reads (nt) }\end{array}$ & $\begin{array}{l}\text { Clean } \\
\text { data (Gb) }\end{array}$ & $\begin{array}{l}\text { Q20 } \\
\text { percentage }\end{array}$ & $\begin{array}{l}\text { Q30 } \\
\text { percentage }\end{array}$ & $\begin{array}{l}\text { GC } \\
\text { percentage }\end{array}$ & $\begin{array}{l}\text { Total mapping } \\
\text { rate }\end{array}$ & $\begin{array}{l}\text { Exon mapping } \\
\text { rate }\end{array}$ & $\begin{array}{l}\text { Intron } \\
\text { mapping rate }\end{array}$ & $\begin{array}{l}\text { Intergenic } \\
\text { mapping rate }\end{array}$ \\
\hline YP1_1 & $25,695,596$ & $25,307,873$ & $7.59 \mathrm{~Gb}$ & $98.34 \%$ & $94.97 \%$ & $42.86 \%$ & $96.86 \%$ & $92.52 \%$ & $3.04 \%$ & $4.44 \%$ \\
\hline YP1_2 & $23,961,907$ & $23,550,226$ & $7.07 \mathrm{~Gb}$ & $97.64 \%$ & $93.25 \%$ & $42.59 \%$ & $96.28 \%$ & $91.83 \%$ & $3.50 \%$ & $4.67 \%$ \\
\hline YP1_3 & $24,090,997$ & $23,756,749$ & 7.13Gb & $98.29 \%$ & $94.86 \%$ & $42.99 \%$ & $96.91 \%$ & $92.81 \%$ & $2.63 \%$ & $4.57 \%$ \\
\hline YP2_1 & $22,458,101$ & $22,064,401$ & $6.62 \mathrm{~Gb}$ & $98.17 \%$ & $94.55 \%$ & $42.76 \%$ & $96.62 \%$ & $91.86 \%$ & $3.51 \%$ & $4.63 \%$ \\
\hline YP2_2 & $26,322,924$ & $25,933,325$ & $7.78 \mathrm{~Gb}$ & $98.35 \%$ & $94.97 \%$ & $42.59 \%$ & $96.82 \%$ & $92.08 \%$ & $3.38 \%$ & $4.54 \%$ \\
\hline YP2_3 & $28,597,224$ & $28,207,492$ & $8.46 \mathrm{~Gb}$ & $98.41 \%$ & $95.20 \%$ & $42.69 \%$ & $96.79 \%$ & $92.28 \%$ & $3.20 \%$ & $4.52 \%$ \\
\hline YP3_1 & $31,454,462$ & $30,971,613$ & $9.29 \mathrm{~Gb}$ & $98.38 \%$ & $95.09 \%$ & $42.53 \%$ & $96.88 \%$ & $91.66 \%$ & $3.60 \%$ & $4.74 \%$ \\
\hline YP3_2 & $24,607,612$ & $24,122,774$ & $7.24 \mathrm{~Gb}$ & $98.40 \%$ & $95.15 \%$ & $42.66 \%$ & $96.74 \%$ & $92.41 \%$ & $3.38 \%$ & $4.21 \%$ \\
\hline YP3_3 & $28,119,471$ & $27,654,238$ & $8.30 \mathrm{~Gb}$ & $98.30 \%$ & $94.91 \%$ & $42.64 \%$ & $96.8 \%$ & $91.74 \%$ & $3.51 \%$ & $4.75 \%$ \\
\hline YP4_1 & $26,766,950$ & $26,483,040$ & $7.94 \mathrm{~Gb}$ & $97.99 \%$ & $93.86 \%$ & $42.82 \%$ & $96.88 \%$ & $92.44 \%$ & $2.94 \%$ & $4.62 \%$ \\
\hline YP4_2 & $27,739,079$ & $27,271,759$ & $8.18 \mathrm{~Gb}$ & $98.21 \%$ & $94.65 \%$ & $42.55 \%$ & $96.5 \%$ & $91.63 \%$ & $3.75 \%$ & $4.62 \%$ \\
\hline YP4_3 & $27,486,903$ & $27,022,147$ & $8.11 \mathrm{~Gb}$ & $98.34 \%$ & $94.99 \%$ & $42.80 \%$ & $96.83 \%$ & $92.15 \%$ & $3.38 \%$ & $4.47 \%$ \\
\hline P1_1 & $24,810,744$ & $24,372,086$ & $7.31 \mathrm{~Gb}$ & $98.22 \%$ & $94.70 \%$ & $42.69 \%$ & $96.67 \%$ & $91.33 \%$ & $3.55 \%$ & $5.11 \%$ \\
\hline P1_2 & $26,317,484$ & $25,820,278$ & $7.75 \mathrm{~Gb}$ & $98.34 \%$ & $95.01 \%$ & $42.15 \%$ & $96.9 \%$ & $91.97 \%$ & $1.87 \%$ & $6.17 \%$ \\
\hline P1_3 & $25,737,276$ & $25,343,570$ & $7.60 \mathrm{~Gb}$ & $98.08 \%$ & $94.30 \%$ & $42.69 \%$ & $96.61 \%$ & $91.70 \%$ & $3.34 \%$ & $4.96 \%$ \\
\hline P2_1 & $25,958,754$ & $25,582,291$ & $7.67 \mathrm{~Gb}$ & $98.20 \%$ & $94.68 \%$ & $42.67 \%$ & $96.24 \%$ & $91.62 \%$ & $3.48 \%$ & $4.90 \%$ \\
\hline P2_2 & $25,124,353$ & $24,744,388$ & $7.42 \mathrm{~Gb}$ & $98.30 \%$ & $94.90 \%$ & $42.87 \%$ & $96.89 \%$ & $92.12 \%$ & $3.23 \%$ & $4.65 \%$ \\
\hline P2_3 & $25,010,070$ & $24,633,309$ & $7.39 \mathrm{~Gb}$ & $98.09 \%$ & $94.39 \%$ & $42.77 \%$ & $96.75 \%$ & $91.87 \%$ & $2.95 \%$ & $5.19 \%$ \\
\hline
\end{tabular}

genes were preferentially expressed in YP1, YP2, YP3 and YP4, respectively, with $\mathrm{P} 1$ and $\mathrm{P} 2$ harboring relative higher numbers (1467 and 779) (Fig. 2), suggesting more distinct transcriptional activities occurred at P1 and P2 stages than at other stages.

\section{Differentially expressed genes at different stages}

Differential expression pattern analysis was performed using DESeq2 [50] R package (1.16.1) with the threshold of adjusted $P$ value $<0.05$. Through pairwise comparison, in total we identified 21,610 DEGs (Additional file 4: Table S2). Clustering analysis of these DEGs based on the FPKM values demonstrated that gene expression models in YP1 to YP4 inflorescence tissues were categorized into one major clade, which was distinguished from another clade consisting of P1 and P2 samples (Fig. 3). The numbers of DEGs between different groups varied from 145 to 13,356 , where comparison between P2 and YP1 yielded the highest whereas that between YP4 and YP3 yielded the lowest number (Additional file 4: Table S2). Moreover, we observed that more temporally separated inflorescences samples yielded more DEGs, suggesting the transcriptional events change dramatically as development duration increased. We inspected the gene expression levels at different continuous intervals, and found comparisons between YP2 and YP1, P1 and YP4 generated more DEGs (2441 for YP2 vs YP1, 8335 for P1 vs YP4) than that in other comparisons (691 for YP3 vs YP2, 145 for YP4 vs YP3, 649 for P2 vs P1) (Additional file 4:
Table S2), indicating more variation in gene expression at the early and late reproductive periods.

Furthermore, we performed GO and KEGG assignments to clarify the potential functions of DEGs at different intervals. According to the GO enrichment results, the up-regulated genes in YP2-vs-YP1 comparison were overrepresented in eleven GO items, including "carbohydrate biosynthetic process" and "protein heterodimerization activity" (Additional file 5: Table S3). In contrast, the down-regulated genes were significantly enriched in sixty-six items, such as "photosynthesis", "translation", "photosynthetic membrane" and "structural constituent of ribosome" (Additional file 5: Table S3). For YP3-vs-YP2 comparison, nineteen GO terms showed highly enrichment, in which "amine metabolic process" and "nucleic acid binding transcription factor activity", and "ribosome" and "protein heterodimerization activity" were highly enriched in up- and down-regulated DEGs (Additional file 5: Table S3), respectively. During YP3 to YP4 phase, thirty-seven terms, such as "cellular carbohydrate metabolic process", "extracellular region", "xyloglucan:xyloglucosyl transferase activity" and "nucleic acid binding transcription factor activity" were enriched in up-regulated DEGs, whereas twentyfive terms containing "cellular component biogenesis" and "nucleosome" significantly appeared in down-regulated DEGs (Additional file 5: Table S3). Classification of DEGs in P1-vs-YP4 comparison yielded the largest number of GO items. The up-regulated genes were abundant 


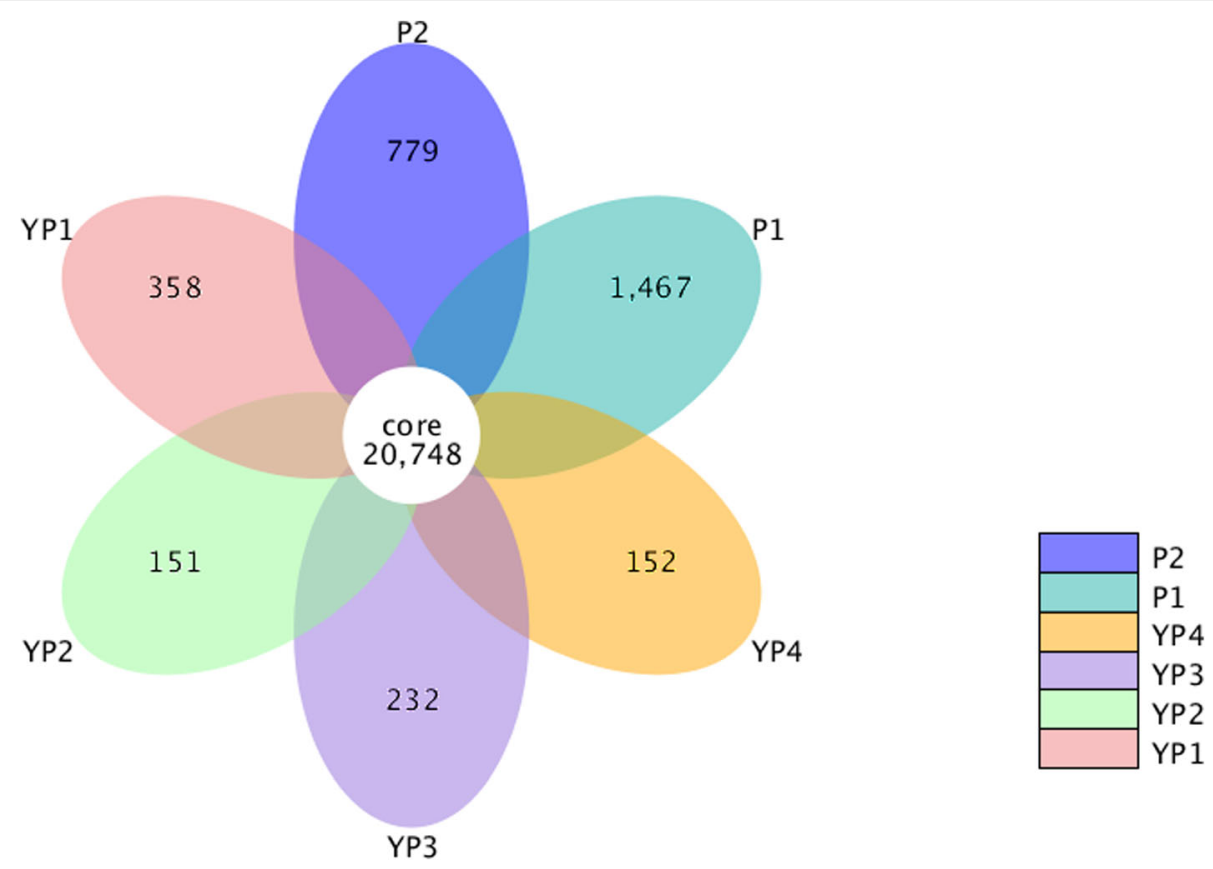

Fig. 2 Venn diagram demonstrating the expressed genes among different-stage inflorescences. The number in core circle means the number of genes expressed at all stage with a cutoff of FPKM $=1$. The numbers in different color ellipses means the preferentially expressed genes with their FKPM $\geq 1$ at one stage but FKPM $<1$ at the other stages

in ninety-two GO terms including "cellular carbohydrate metabolic process", "apoplast" and "oxidoreductase activity, and acting on peroxide as acceptor" (Additional file 5: Table S3). Meanwhile, the down-regulated genes were enriched in forty GO terms which encompassed "DNA replication", "chromosome" and "microtubule motor activity" (Additional file 5: Table S3). For P2-vs$\mathrm{P} 1$, the terms "response to oxidative stress", "apoplast" and "peroxidase activity" were highly represented in upregulated genes while "trehalose biosynthetic process" and "phosphoric ester hydrolase activity" were enriched in down-regulated genes (Additional file 5: Table S3).

The KEGG enrichment analysis results showed that at early stage (in YP2-vs-YP1), a large proportion of upregulated DEGs were enriched in pathways "Plant hormone signal transduction" and "Biosynthesis of secondary metabolites", and on the other hand "Photosynthesis-antenna proteins", "Ribosome" were overrepresented in down-regulated DEGs (Table 2). The DEGs in YP3-vs-YP2 and YP4-vs-YP3 comparisons were enriched in pathways "Diterpenoid biosynthesis", "Flavonoid biosynthesis" and "Ribosome" (Table 2). At late reproductive stage (in P1-vs-YP4), twenty pathways were enriched, out of which "Carbon fixation in photosynthetic organisms", "Phenylpropanoid biosynthesis", "Carbon metabolism", "Nitrogen metabolism" as well as "Diterpenoid biosynthesis" and "Flavonoid biosynthesis" were overrepresented in DEGs with increased expression levels, while "DNA replication" was highly enriched in DEGs with decreased expression levels (Table 2). In P2-vs-P1, we found "Fatty acid elongation" and "Starch and sucrose metabolism" were significantly related to the up- and down-regulated DEGs (Table 2), respectively. The GO and KEGG enrichment results could provide useful cues for deciphering the genes involved in quinoa inflorescence morphogenesis.

\section{Transcription factor dynamics during inflorescence development}

Transcription factors (TFs) are regarded as critical regulators in many plant developmental events. In order to survey the TFs dynamics during all quinoa inflorescence stages, we identified the genome-wide TF encoding genes and the TFs in the DEGs using an online TF prediction tool in Plant Transcription Factor Database v4.0 [51]. As illustrated in Additional file 6: Table S4, in upor down-regulated DEGs at different periods TFs accounted for considerable proportion (approximately 4.26 to $16.67 \%$ ) (Additional file 2: Figure S2, A), which was larger than the genome-wide TFs proportion (4.14\%) (Additional file 7: Table S5). Among all developmental intervals, the phases YP2-vs-YP1 and P1-vs-YP4 have the highest numbers of differentially expressed TFs (Additional file 6: Table S4). One hundred sixty nine TF encoding genes out of 2441 DEGs (accounting for 6.9\%) were identified in YP2-vs-YP1 comparison (Additional file 6: Table S4). By comparing the TF proportion in DEGs with that in the genome background (Additional 


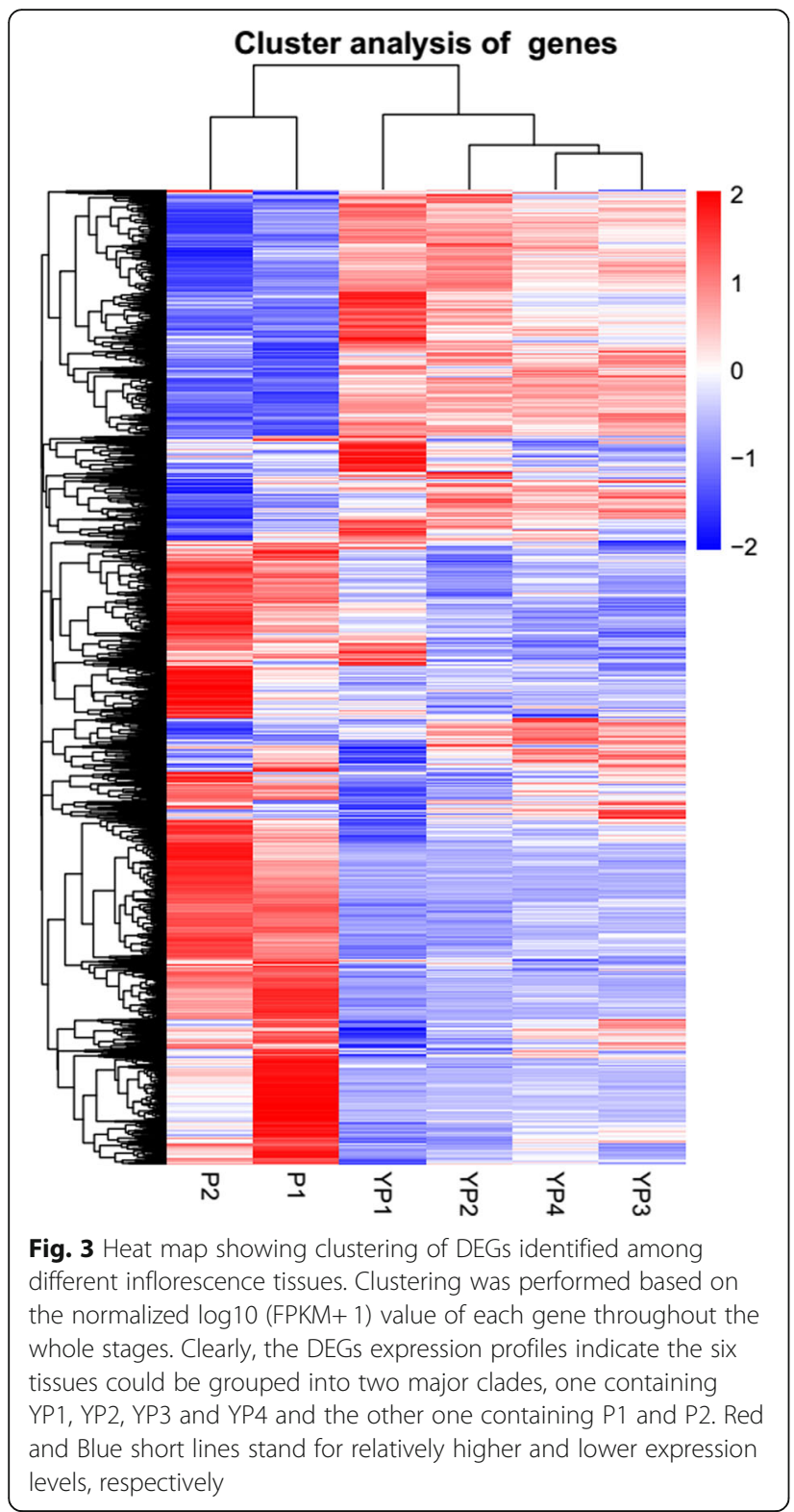

file 2: Figure S2, B), we found B3, MIKC-type MADSbox and $\mathrm{C} 2 \mathrm{H} 2$ family members were highly more enriched in the up-regulated TFs whereas bHLH, ERF (Ethylene-Response Factor), MYB, TCP (TEOSINTE BRANCHED, CYCLOIDEA and PCF), G2-like, GATA and HD-ZIP family members were significantly more abundant in down-regulated TFs (Fig. 4, a-b). In P1-vsYP4 comparison, 270 and 202 TFs encoding genes represented in 5841 up- and 2494 down-regulated DEGs (Additional file 6: Table S4), respectively. In the same way, ERF, NAC, C2H2, bZIP, WRKY, MADS-box (including MIKC-type and M-type MADS), G2-like and LBD encoding genes were highly more enriched in upregulated TFs (Fig. 4c). In contrast, B3, bHLH, GRAS, HD-ZIP, SBP and GATA members were more abandunt in down-regulated DEGs (Fig. 4d). Interestingly, MYB was overrepresented in up-regulated as well as in downregulated DEGs (Fig. 4, c-d). These TFs mentioned above were also identified in YP3-vs-YP2, YP4-vs-YP3 and P2-vs-P1.

In addition, we found several important inflorescence development related TFs expressed in all stages, including LFY, CO-like (CONSTANS-like), WOX (WUSCHEL-related homeobox), TALE, AP2, DOF and YABBY (Additional file 6: Table S4; Fig. 4, a-d). The TFs profiling at different intervals could supply with cues for further investigation of the inflorescence morphology changes.

\section{Weighted gene co-expression network analysis of DEGs}

Weighted Gene Co-Expression Network Analysis (WGCNA) [52] is a powerful tool for generating gene co-expression networks for RNA-seq analysis. Using this tool co-regulated genes that may possess similar biological function could be identified. To uncover the potential regulatory networks regarding to quinoa inflorescence development, we carried out WGCNA on the DEGs. Totally, we found 21,568 out of 21,610 DEGs (99.8\%) across all the inflorescence developmental stages could be categorized into 10 modules (Fig. 5a), with the Turquoise module harboring the most genes (5949 DEGs) whereas the Purple module harboring the least genes (32 DEGs) (Additional file 8: Table S6). Remarkably, the majority of the genes in the Turquoise, Blue, Green, Black, Pink and Purple modules showed highly specific expression pattern at one single stage. As showed in Fig. 5b, the Turquoise and Purple modules were significantly more abundant at P2 stage, the Blue and Black modules were mainly expressed at P1 stage, whereas the Green and Pink modules showed high expression at YP1 stage (Fig. 5b).

To further investigate the biological functions of these gene modules, we performed KEGG analysis. As detailed in Fig. 5c, for the Turquoise module, pathways including "Carbon fixation in photosynthetic organisms", "Carbon metabolism", "Glyoxylate and dicarboxylate metabolism", "Pentose phosphate pathway", "Amino sugar and nucleotide sugar metabolism" and "Biosynthesis of amino acids" were overrepresented (Fig. 5c). The Brown module was abundant in "DNA replication", "Homologous recombination", "Mismatch repair", "Pyrimidine metabolism", "Non-homologous end-joining", "Base excision repair" and "Ribosome biogenesis in eukaryotes" (Fig. 5c). The Yellow module contained most genes enriched in "Ribosome", "Proteasome" and "Spliceosome". The Green module was only highly enriched in "Plant hormone signal transduction" (Fig. 5c). For the Pink module, "Ribosome", "Porphyrin and chlorophyll metabolism", "Photosynthesis" and "Photosynthesis-antenna proteins" were significantly enriched (Fig. 5c). 
Table 2 KEGG classification of the DEGs at different intervals

\begin{tabular}{|c|c|c|c|c|}
\hline & \multicolumn{2}{|l|}{ Up-regulated } & \multicolumn{2}{|l|}{ Down-regulated } \\
\hline & Item & $p$-value & Item & $p$-value \\
\hline \multirow[t]{7}{*}{ YP2-vs-YP1 } & Plant hormone signal transduction & 0.030906063 & Photosynthesis - antenna proteins & 7.75E-08 \\
\hline & \multirow[t]{6}{*}{ Biosynthesis of secondary metabolites } & \multirow[t]{6}{*}{0.035863996} & Ribosome & 4.67E-06 \\
\hline & & & Porphyrin and chlorophyll metabolism & $2.14 \mathrm{E}-05$ \\
\hline & & & Photosynthesis & $2.14 \mathrm{E}-05$ \\
\hline & & & Carbon fixation in photosynthetic organisms & 0.0094376 \\
\hline & & & Nitrogen metabolism & 0.0207042 \\
\hline & & & Arginine biosynthesis & 0.0207042 \\
\hline YP3-vs-YP2 & Diterpenoid biosynthesis & 0.005119967 & Ribosome & $1.92 \mathrm{E}-13$ \\
\hline \multirow[t]{5}{*}{ YP4-vs-YP3 } & Flavonoid biosynthesis & 0.025536677 & Ribosome & 0.0362887 \\
\hline & Nitrogen metabolism & 0.025536677 & \multirow[t]{4}{*}{ Sulfur metabolism } & \multirow[t]{4}{*}{0.0362887} \\
\hline & Ubiquinone and other terpenoid-quinone biosynthesis & 0.025536677 & & \\
\hline & Phenylalanine metabolism & 0.025536677 & & \\
\hline & Phenylpropanoid biosynthesis & 0.049009994 & & \\
\hline \multirow[t]{15}{*}{ P1-vs-YP4 } & Carbon fixation in photosynthetic organisms & 0.000192461 & DNA replication & $2.28 \mathrm{E}-05$ \\
\hline & Phenylpropanoid biosynthesis & 0.000192461 & Mismatch repair & 0.0059843 \\
\hline & Carbon metabolism & 0.000818142 & Purine metabolism & 0.0190094 \\
\hline & Nitrogen metabolism & 0.001083904 & Pyrimidine metabolism & 0.0194897 \\
\hline & Glyoxylate and dicarboxylate metabolism & 0.003257291 & \multirow[t]{11}{*}{ Homologous recombination } & \multirow[t]{11}{*}{0.0215846} \\
\hline & Starch and sucrose metabolism & 0.00366431 & & \\
\hline & Plant-pathogen interaction & 0.004811801 & & \\
\hline & Photosynthesis - antenna proteins & 0.005158419 & & \\
\hline & Alanine, aspartate and glutamate metabolism & 0.016187972 & & \\
\hline & Photosynthesis & 0.020905938 & & \\
\hline & Diterpenoid biosynthesis & 0.026926862 & & \\
\hline & Flavonoid biosynthesis & 0.034576313 & & \\
\hline & Pentose and glucuronate interconversions & 0.034576313 & & \\
\hline & Phenylalanine metabolism & 0.04782332 & & \\
\hline & Fructose and mannose metabolism & 0.04782332 & & \\
\hline \multirow[t]{2}{*}{ P2-vs-P1 } & \multirow[t]{2}{*}{ Fatty acid elongation } & \multirow[t]{2}{*}{0.048695132} & Starch and sucrose metabolism & 0.0019501 \\
\hline & & & Galactose metabolism & 0.048367 \\
\hline
\end{tabular}

DEGs were subjected to KEGG enrichment analysis using cluster Profiler R package. Items with $P$ value less than 0.05 were considered significantly enriched

\section{Identification of the core transcriptional regulators modulating inflorescence architecture}

Gene sets that show highly stage-specific expression patterns usually are closely related to the corresponding phenotypes. To investigate the regulatory networks shaping inflorescence architecture, we intensively focused on the modules including Green and Pink with high specific expression at early reproductive phase panicles (none branching stage), and the modules including Turquoise, Blue, Black, and Purple with high specific expression at late reproductive phase panicles (branching stage). As TFs play essential roles for the morphogenesis of various plant tissues and organs, we sorted out the TF encoding genes of each module. Further, we generated core transcriptional regulatory networks of each module using the TFs (Additional file 9: Table S7) and key homologs (Additional file 10: Table S8) involved in inflorescence architecture regulation as seed nodes.

Totally, with the exception that Purple harbored none TFs, 48 different TF families were identified among these modules (Additional file 9: Table S7). The Turquoise module harbored the largest TF number (300), and the Green module harbored the highest TF proportion (7.57\%) (Additional file 9: Table S7, Fig. 6a). Obviously, the TF percentages of these modules were larger than that in the genome background with the exception of the Pink module $(2.72 \%$ vs $4.14 \%)$ (Fig. 6a). Notably, some TFs were only present in one module, 


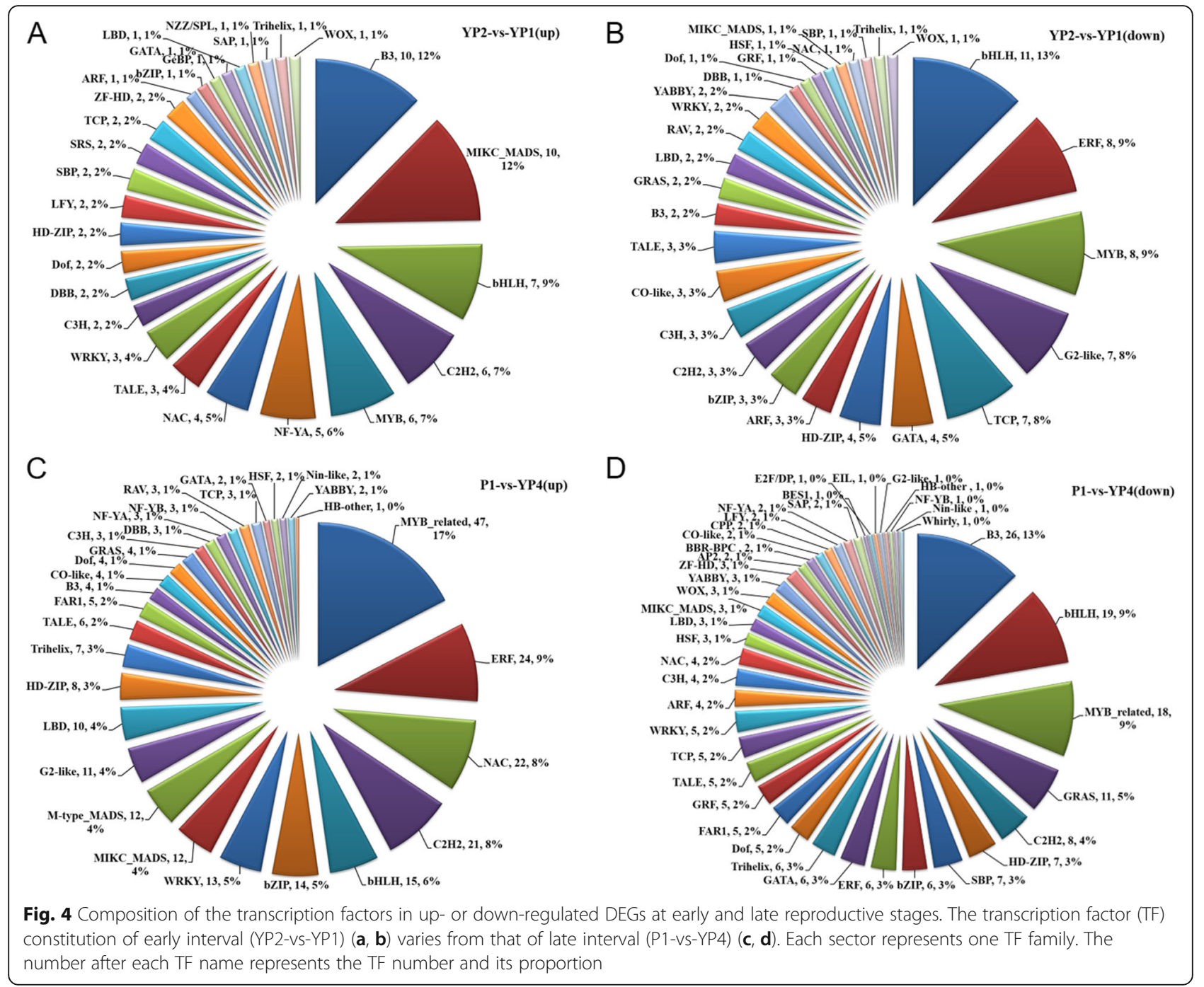

such as LFY (Gene ID: 110698679) in the Pink module, ZF-HD (Gene ID: 110717138) and GRF (Gene ID: 110738890) in the Green module, and VOZ (Gene ID: 110693688), STAT (Gene ID: 110694069), SRS (Gene ID: 110723239, 110,724,730, novel.32919), NF-YA (Gene ID: 110684358, 110,737,411, novel.26441), GeBP (Gene ID: $110687270,110,693,452)$, CAMTA (Gene ID: 110697210) and BBR-BPC (Gene ID: 110686586, 110, 692,413 ) in the Turquoise module (Fig. 6b). Further, we found the TF composition varied among different modules. For instance, bHLH is the largest TF family in the Turquoise module; the Blue module harbors the most MYB members; MADS tops in the Green and Pink modules; while $\mathrm{C} 3 \mathrm{H}$ tops in the Black module (Fig. 6b). The module-specific TF composition may render special effects for inflorescence development.

In order to screen the principle regulators participating in maintaining inflorescence morphology at different stages, we manually refined the corresponding modules with proper edge weight cutoffs and visualized each core networks using Cytoscape [53]. As detailed in Table 3, we found the central hubs in modules related to early reproductive phase inflorescences significantly varied from that in modules related to late reproductive phase inflorescences. For the Green module, a core network containing 602 nodes and 2253 edges were obtained. Using Cytoscape we filtered to obtain the largest 20 hubs with top degrees, among which 14 were TF encoding genes (Fig. 7a, Table 3). Specifically, the largest hub 110, 723,560 is a B3 TF. $110,692,073$ encodes a WUSCHELrelated homeobox (WOX) protein which is important for floral meristem development in Arabidopsis [16] (Fig. 7a). 110,715,387 and 110,705,456 are TALE family members that share homeodomain with KNOX and WOX proteins. 110,717,078 and 110,682,413 encode GRAS TFs resembling the rice MONOCULM 1 (MOC1, [54]) that controls tiller number and panicle branches. $110,708,326$ encodes a LSH protein belonging to the 

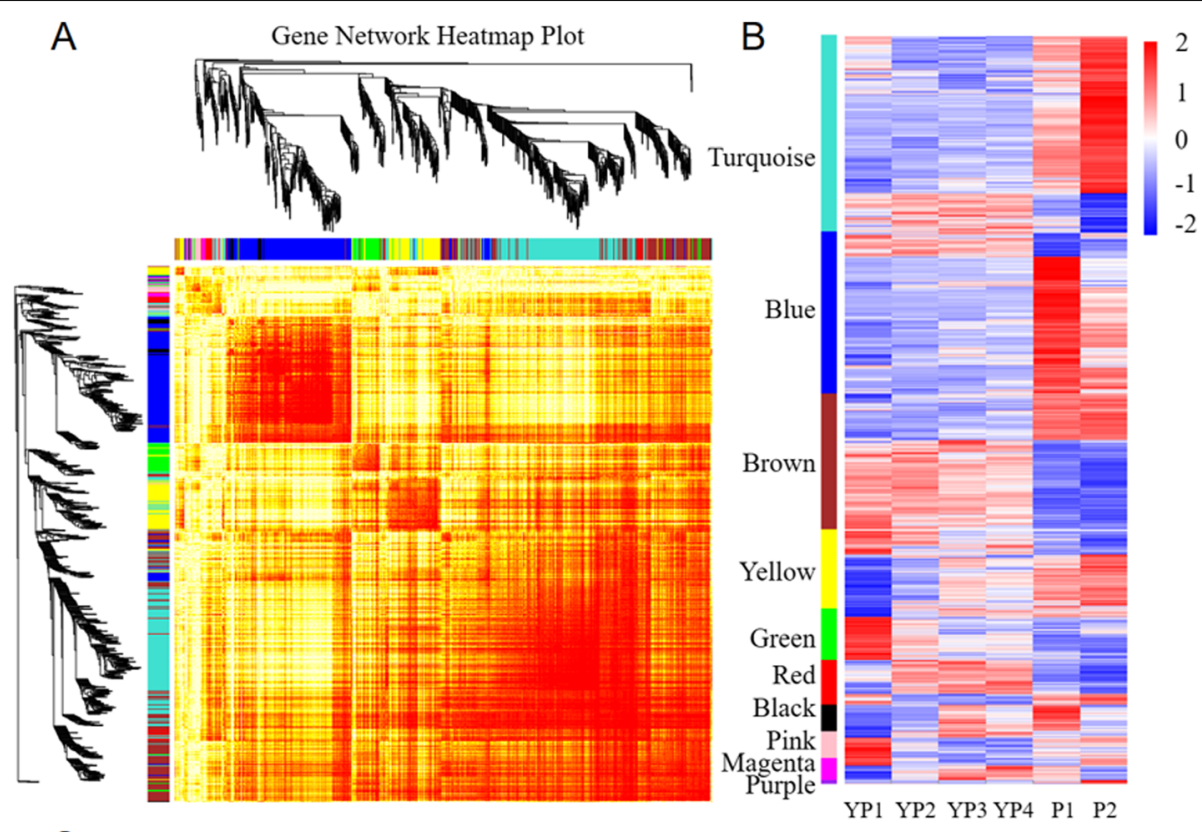

C

\begin{tabular}{|c|c|c|c|c|c|}
\hline Modules & KEGG ID & Description & pvalue & padj & Count \\
\hline & ath00710 & Carbon fixation in photosynthetic organisms & $3.41 \mathrm{E}-08$ & $4.06 \mathrm{E}-06$ & 22 \\
\hline & ath01200 & Carbon metabolism & $5.28 \mathrm{E}-07$ & 3.14E-05 & 47 \\
\hline & ath00630 & Glyoxylate and dicarboxylate metabolism & 0.0002408 & 0.0077534 & 16 \\
\hline Turquoise & ath00030 & Pentose phosphate pathway & 0.0002627 & 0.0077534 & 14 \\
\hline & ath00520 & Amino sugar and nucleotide sugar metabolism & 0.0003258 & 0.0077534 & 22 \\
\hline & ath01230 & Biosynthesis of amino acids & 0.001024 & 0.0203086 & 33 \\
\hline & ath03030 & DNA replication & $1.35 \mathrm{E}-08$ & $1.25 \mathrm{E}-06$ & 16 \\
\hline & ath03440 & Homologous recombination & 3.34E-05 & 0.0015544 & 13 \\
\hline & ath03430 & Mismatch repair & $6.98 \mathrm{E}-05$ & 0.0021641 & 10 \\
\hline Brown & ath00240 & Pyrimidine metabolism & 0.0018918 & 0.0417514 & 16 \\
\hline & ath03450 & Non-homologous end-joining & 0.0022447 & 0.0417514 & 4 \\
\hline & ath03410 & Base excision repair & 0.003214 & 0.0489872 & 7 \\
\hline & ath03008 & Ribosome biogenesis in eukaryotes & 0.0036872 & 0.0489872 & 11 \\
\hline & ath03010 & Ribosome & $8.50 \mathrm{E}-40$ & $6.46 \mathrm{E}-38$ & 83 \\
\hline Yellow & ath03050 & Proteasome & $2.83 \mathrm{E}-06$ & 0.0001076 & 14 \\
\hline & ath03040 & Spliceosome & 0.0001638 & 0.0041494 & 21 \\
\hline Green & ath04075 & Plant hormone signal transduction & 0.0003586 & 0.0254578 & 14 \\
\hline & ath03010 & Ribosome & $2.77 \mathrm{E}-06$ & $9.13 \mathrm{E}-05$ & 15 \\
\hline & ath00860 & Porphyrin and chlorophyll metabolism & 0.0002757 & 0.0045494 & 5 \\
\hline & ath00195 & Photosynthesis & 0.0014875 & 0.016362 & 6 \\
\hline & ath00196 & Photosynthesis - antenna proteins & 0.0021663 & 0.0178721 & 3 \\
\hline
\end{tabular}

Fig. 5 Weighted gene co-expression network analysis of DEGs. a 21,568 out of 21,610 DEGs are grouped into ten gene modules. 5949, 5410, 4442, 2206, 1532, 836, 417, 404, 340 and 32 DEGs were co-expressed in the Turquoise, Blue, Brown, Yellow, Green, Red, Black, Pink, Magenta and Purple modules, respectively. $\mathbf{b}$ Heat map showing the gene expression levels of DEGs in different modules. $\mathbf{c}$ KEGG enrichment analysis of different gene sets

same ALOG family with spikelet number regulator TAW1 [35]. 110,700,571 encodes a plant-specific TCP, resembling the maize TB [55], the rice OsTB1 and the homologous BRANCHED1 (BRC1) proteins in Arabidopsis [56], pea and tomato, which have conserved roles in suppressing branching. Besides, the core hubs also contained WRKY (Gene ID: 110710092 and 110,682, 067), bHLH (Gene ID: 110714201 and 110,703,894), HSF (Gene ID: 110686357) and EIL (Gene ID: 110695891) (Fig. 7a). In another early reproductive phase related Pink module, we found 11 central hub genes were TFs in the refined core network (Fig. 7b). Especially, the largest hub encodes a LFY protein (110698679) (Fig. 7b) whose homologs have been widely identified as crucial floral identity genes in many other plant species $[3,57]$. The second largest hub is $110,729,369$, encoding a Trihelix protein. 110,736,045 and novel.22515 encode an M-type (MY) and MICK-type (PISTILLATA-like) MADSbox protein, respectively, which are crucial for floral development [58]. Meanwhile, several plant development related genes, such as DOF (Gene ID: 110711979), G2-like (Gene ID: 110691257), HD-ZIP (Gene ID: 110729692) and RAV (Gene ID: 110719982), B3 (Gene ID: novel.9281), $M Y B$ (Gene ID: 110708638) and YABBY (Gene ID: 110685646) were also identified as hub genes in this module (Fig. 7b). 


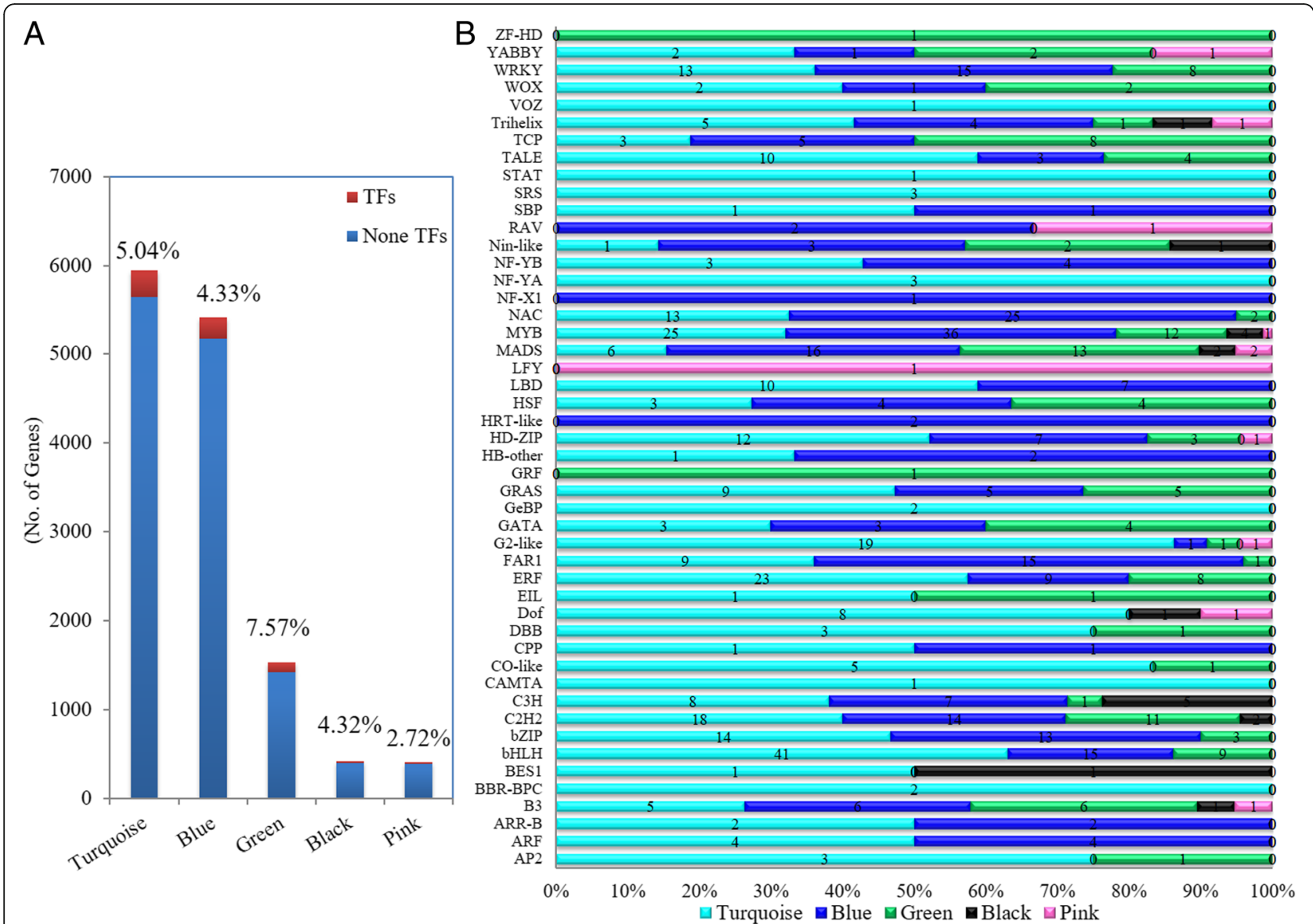

Fig. 6 Distribution of transcription factors in Turquoise, Blue, Green, Black and Pink module. a Statistic result of the TFs number and its proportion at each gene module. $\mathbf{b}$ the composition of TF family members distributed at different gene modules

Subsequently, we analyzed the modules closely related to late reproductive phase panicles. In the Blue module, the largest hub is a $M Y B$ gene (Gene ID: novel.11405) (Fig. 7c). Surprisingly, 5 out of 20 hubs are revealed to be MADS-box genes including one MICK-type (AGL18like) (Gene ID: 110707171) and four M-type $(M \alpha)$ (Gene ID: $110685095,110,735,861,110,740,130,110,736,081)$ (Fig. 7c). Besides, we found co-regulated genes including C2H2 (Gene ID: 110688475, 110,684,112, 110,737,201), LBD (Gene ID: 110696735), bZIP (Gene ID: 110697372) and NAC (Gene ID: 110726622 and 110,726,634) are specifically present as hubs in the Blue module rather than in other modules (Fig. 7c). In the Black module, the M-type $(M \beta)$ MADS-box gene (Gene ID: novel.4708) harbors the most edges (Fig. 7d). Another MADS-box gene (Gene ID: 110725731), a homolog of the Arabidopsis PISTILLATA which is homeotic B-class gene involved in petal and stamen specification [58], was also present as a hub gene in this module (Fig. 7d). Interestingly, several C3H genes (Gene ID: novel.34399, 110,698,972, 110,736,053 and novel.34398) and a Nin-like (Gene ID: novel.40662) gene were identified as the specific central hubs in this module (Fig. 7d). In the core subnetwork of the Turquoise module, the largest two hubs 110,697,084 and $110,729,689$ (Fig. 7e) were homologous to TFL which interacts with $A P 1$ and $L F Y$ to control floral transition as well as plant inflorescence architecture in many plants [3, 4]. 110,694,654 and 110,733,648 encode ERF TFs whose counterparts regulate floral meristem specification in Arabidopsis and rice. Remarkably, a MICKtype MADS-box encoding gene (Gene ID: 110722241), a homolog of the Arabidopsis FRUITFULL which controls meristem identity and fruit development [58], appeared as another hub in this module (Fig. 7e). Meanwhile, $H D$ ZIP (Gene ID: 110722837, 110,722,774, 110,731,701), FAR1 (Gene ID: 110722691) as well as TCP (Gene ID: 110694654) genes were also included in this late reproductive stage associated module (Fig. 7e).

\section{Real-time validation of RNA-seq data}

To test the reliability of RNA-Seq data, we performed real-time PCR to measure the expression levels of five genes at six stages, including CqAPO1-1 (Gene ID: 110686960), CqAPO1-2 (Gene ID: 110737497), CqTFL7 
Table 3 List of transcription factors with top degrees in the core network of each module

\begin{tabular}{|c|c|c|c|c|c|}
\hline Module & Gene ID & Gene name & Degree & Best hit in A.thaliana & Description for the best hit \\
\hline \multirow[t]{14}{*}{ Green } & $110,723,560$ & B3 & 586 & AT5G42700.1 & B3 family protein \\
\hline & $110,715,387$ & TALE & 355 & AT4G32980.1 & homeobox gene 1 \\
\hline & $110,705,456$ & TALE & 283 & AT4G32980.1 & homeobox gene 1 \\
\hline & $110,717,078$ & GRAS & 245 & AT3G49950.1 & GRAS family protein \\
\hline & $110,682,413$ & GRAS & 184 & AT3G49950.1 & GRAS family protein \\
\hline & $110,708,326$ & $\mathrm{LSH} 4$ & 179 & AT3G23290.2 & $\begin{array}{l}\text { LIGHT-DEPENDENT SHORT } \\
\text { HYPOCOTYLS-like protein }\end{array}$ \\
\hline & $110,710,092$ & WRKY & 76 & AT5G43290.1 & WRKY DNA-binding protein 49 \\
\hline & $110,682,067$ & WRKY & 36 & AT4G39410.1 & WRKY DNA-binding protein 13 \\
\hline & $110,714,201$ & $\mathrm{bHLH}$ & 33 & AT4G37850.1 & bHLH family protein \\
\hline & $110,692,073$ & WOX & 29 & AT3G18010.1 & WUSCHEL related homeobox 1 \\
\hline & $110,703,894$ & $\mathrm{bHLH}$ & 27 & AT3G26744.1 & bHLH family protein \\
\hline & $110,686,357$ & HSF & 27 & AT1G46264.1 & heat shock transcription factor B4 \\
\hline & $110,695,891$ & EIL & 25 & AT5G65100.1 & EIL family protein \\
\hline & $110,700,571$ & $\mathrm{TCP}$ & 22 & AT3G47620.1 & $\begin{array}{l}\text { TEOSINTE BRANCHED, cycloidea } \\
\text { and PCF (TCP) } 14\end{array}$ \\
\hline \multirow[t]{11}{*}{ Pink } & $110,698,679$ & LFY & 57 & AT5G61850.1 & $\begin{array}{l}\text { floral meristem identity control } \\
\text { protein LEAFY (LFY) }\end{array}$ \\
\hline & $110,729,369$ & Trihelix & 55 & AT5G03680.1 & Trihelix family protein \\
\hline & $110,729,692$ & $\mathrm{HD}-\mathrm{ZIP}$ & 47 & AT4G00730.1 & HD-ZIP family protein \\
\hline & $110,719,982$ & RAV & 46 & AT1G13260.1 & related to $A B \mid 3 / N P 11$ \\
\hline & $110,691,257$ & G2-like & 44 & AT1G25550.1 & G2-like family protein \\
\hline & $110,736,045$ & M-type_MADS & 33 & AT5G48670.1 & AGAMOUS-like 80 \\
\hline & novel.9281 & B3 & 29 & AT4G33280.1 & B3 family protein \\
\hline & $110,708,638$ & MYB_related & 28 & AT3G21430.2 & DNA binding \\
\hline & $110,685,646$ & YABBY & 25 & AT1G69180.1 & YABBY family protein \\
\hline & novel.22515 & MIKC_MADS & 16 & AT5G20240.1 & MIKC_MADS family protein \\
\hline & $110,711,979$ & Dof & 14 & AT2G28510.1 & Dof family protein \\
\hline \multirow[t]{15}{*}{ Blue } & novel.11405 & MYB_related & 316 & AT4G09450.1 & MYB_related family protein \\
\hline & $110,707,171$ & MIKC_MADS & 282 & AT5G13790.1 & AGAMOUS-like 15 \\
\hline & $110,688,475$ & $\mathrm{C} 2 \mathrm{H} 2$ & 258 & AT5G59820.1 & $\mathrm{C} 2 \mathrm{H} 2$ family protein \\
\hline & $110,684,112$ & $\mathrm{C} 2 \mathrm{H} 2$ & 237 & AT5G59820.1 & $\mathrm{C} 2 \mathrm{H} 2$ family protein \\
\hline & $110,685,095$ & M-type_MADS & 214 & AT5G60440.1 & AGAMOUS-like 62 \\
\hline & novel.23507 & MYB_related & 176 & AT5G04760.1 & MYB family protein \\
\hline & $110,735,861$ & M-type_MADS & 119 & AT5G60440.1 & AGAMOUS-like 62 \\
\hline & $110,740,130$ & M-type_MADS & 75 & AT2G24840.1 & AGAMOUS-like 61 \\
\hline & $110,696,735$ & LBD & 36 & AT3G49940.1 & LOB domain-containing protein 38 \\
\hline & $110,736,081$ & M-type_MADS & 34 & AT5G60440.1 & AGAMOUS-like 62 \\
\hline & $110,737,201$ & $\mathrm{C} 2 \mathrm{H} 2$ & 25 & AT5G56200.1 & $\mathrm{C} 2 \mathrm{H} 2$ family protein \\
\hline & $110,697,372$ & bZIP & 19 & AT3G62420.1 & basic region/leucine zipper motif 53 \\
\hline & $110,726,622$ & NAC & 15 & AT3G04070.1 & NAC domain containing protein 47 \\
\hline & $110,726,634$ & NAC & 15 & AT3G04070.1 & NAC domain containing protein 47 \\
\hline & $110,719,209$ & RAV & 14 & AT1G50680.1 & RAV family protein \\
\hline \multirow[t]{2}{*}{ Black } & novel.4708 & M-type_MADS & 223 & AT5G55690.1 & M-type_MADS family protein \\
\hline & novel.40662 & Nin-like & 181 & AT2G43500.1 & Nin-like family protein \\
\hline
\end{tabular}


Table 3 List of transcription factors with top degrees in the core network of each module (Continued)

\begin{tabular}{|c|c|c|c|c|c|}
\hline Module & Gene ID & Gene name & Degree & Best hit in A.thaliana & Description for the best hit \\
\hline & $110,711,872$ & Trihelix & 180 & AT2G33550.1 & Trihelix family protein \\
\hline & $110,687,197$ & MYB_related & 128 & AT5G04760.1 & MYB family protein \\
\hline & novel.34399 & $\mathrm{C} 3 \mathrm{H}$ & 106 & AT3G19360.1 & $\mathrm{C} 3 \mathrm{H}$ family protein \\
\hline & $110,698,972$ & $\mathrm{C} 3 \mathrm{H}$ & 93 & AT3G19360.1 & $\mathrm{C} 3 \mathrm{H}$ family protein \\
\hline & $110,736,053$ & $\mathrm{C} 3 \mathrm{H}$ & 58 & AT1G03790.1 & $\mathrm{C} 3 \mathrm{H}$ family protein \\
\hline & novel.34398 & $\mathrm{C} 3 \mathrm{H}$ & 50 & AT3G19360.1 & $\mathrm{C} 3 \mathrm{H}$ family protein \\
\hline & $110,725,731$ & MIKC_MADS & 10 & AT5G20240.1 & MIKC_MADS family protein \\
\hline \multirow[t]{10}{*}{ Turquoise } & $110,697,084$ & TFL & 85 & AT5G03840.1 & TERMINAL FLOWER 1 \\
\hline & $110,729,689$ & TFL & 44 & AT5G03840.1 & TERMINAL FLOWER 1 \\
\hline & $110,722,691$ & FAR1 & 18 & AT1G10240.1 & FAR1-related sequence 11 \\
\hline & $110,722,837$ & $\mathrm{HD}-\mathrm{ZIP}$ & 18 & AT5G47370.1 & $\begin{array}{l}\text { Homeobox-leucine zipper protein } 4 \\
\text { (HB-4) / HD-ZIP protein }\end{array}$ \\
\hline & $110,694,654$ & TCP & 18 & AT3G27010.1 & TCP family protein \\
\hline & $110,733,648$ & ERF & 17 & AT5G25190.1 & ERF family protein \\
\hline & $110,711,921$ & ERF & 16 & AT5G13330.1 & related to AP2 6I \\
\hline & $110,722,774$ & HD-ZIP & 15 & AT3G61890.1 & homeobox 12 \\
\hline & $110,731,701$ & $\mathrm{HD}-\mathrm{ZIP}$ & 14 & AT2G46680.1 & homeobox 7 \\
\hline & $110,722,241$ & MIKC_MADS & 14 & AT5G60910.1 & AGAMOUS-like 8 \\
\hline
\end{tabular}

The detailed information of the central TF hubs in Fig.7 is displayed in this table. The homologs of these hubs were identified in model plant Arabidopsis thaliana based on the prediction results obtained in Plant Transcription Factor Database v4.0 (http://planttfdb.cbi.pku.edu.cn)

(Gene ID: 110697084), CqFTL5 (Gene ID: 110701053) and CqFTL9 (Gene ID: 110697999). Then we normalized and compared the real-time PCR results with that of RNA-Seq data. We found the gene expression levels measured by real-time PCR were highly consistent with that obtained from RNA-Seq analysis (Fig. 8), suggesting the high quality of the transcriptome data.

\section{Discussion}

Improvement of inflorescence or panicle structure could effectively increase the grain yield and thus it is an important rational designing concept [5]. Over past decades, the key advances in the model plant Arabidopsis, in grasses as well as in legumes have shed lights on how the biological events including floral transition, panicle branching and spikelet formation interact with each other and contribute to the final yield. Though many regulators act in conserved manners among different plant species, however, divergent nodes still exist which may be responsible for the distinct inflorescence architectures [3]. Therefore, detailed investigation should be conducted especially for poorly understood plants. Quinoa is an important high-nutritional pseudo cereal, but maximizing its yield potential is still under progress to meet the worldwide demands [45]. In the present study, we conducted comprehensive investigation on quinoa inflorescence development, and generated regulatory networks patterning early reproductive phase and late reproductive phase inflorescence architecture using
RNA-seq and Weighted Gene Co-Expression Network Analysis (WGCNA) methods.

We performed transcriptomic analysis using the quinoa inflorescence samples collected from six stages after floral transition. Pearson correlation analysis and clustering of the differentially expressed genes (DEGs) indicated the developmental activities in YP1, YP2, YP3 and YP4 samples resembled each other but were divergent from P1 and P2 samples. KEGG analysis of the DEGs at early reproductive intervals yield distinct pathway items compared with that yielded at late intervals. For example, the up- and down-regulated DEGs between YP2 and YP1 were enriched in "Plant hormone signal transduction" and "Photosynthesis", respectively. By contrast, the up- and down-regulated DEGs between P1 and YP4 were overrepresented in "Carbon fixation in photosynthetic organisms" and "DNA replication", respectively. These pathway differences were accompanied with transcriptional activities variation which was reflected by the transcription factors (TFs) composition of DEGs at different intervals. Thus, characterization of these different-stage transcriptomes could facilitate identifying the potential key regulators involved in inflorescence architecture formation. However, large-scale transcriptome analysis usually yields a great deal of DEGs, increasing the difficult in screening potential candidates. Besides, inflorescence development processes are usually controlled by a great complex of regulators, hence identification of the hub genes demands very 


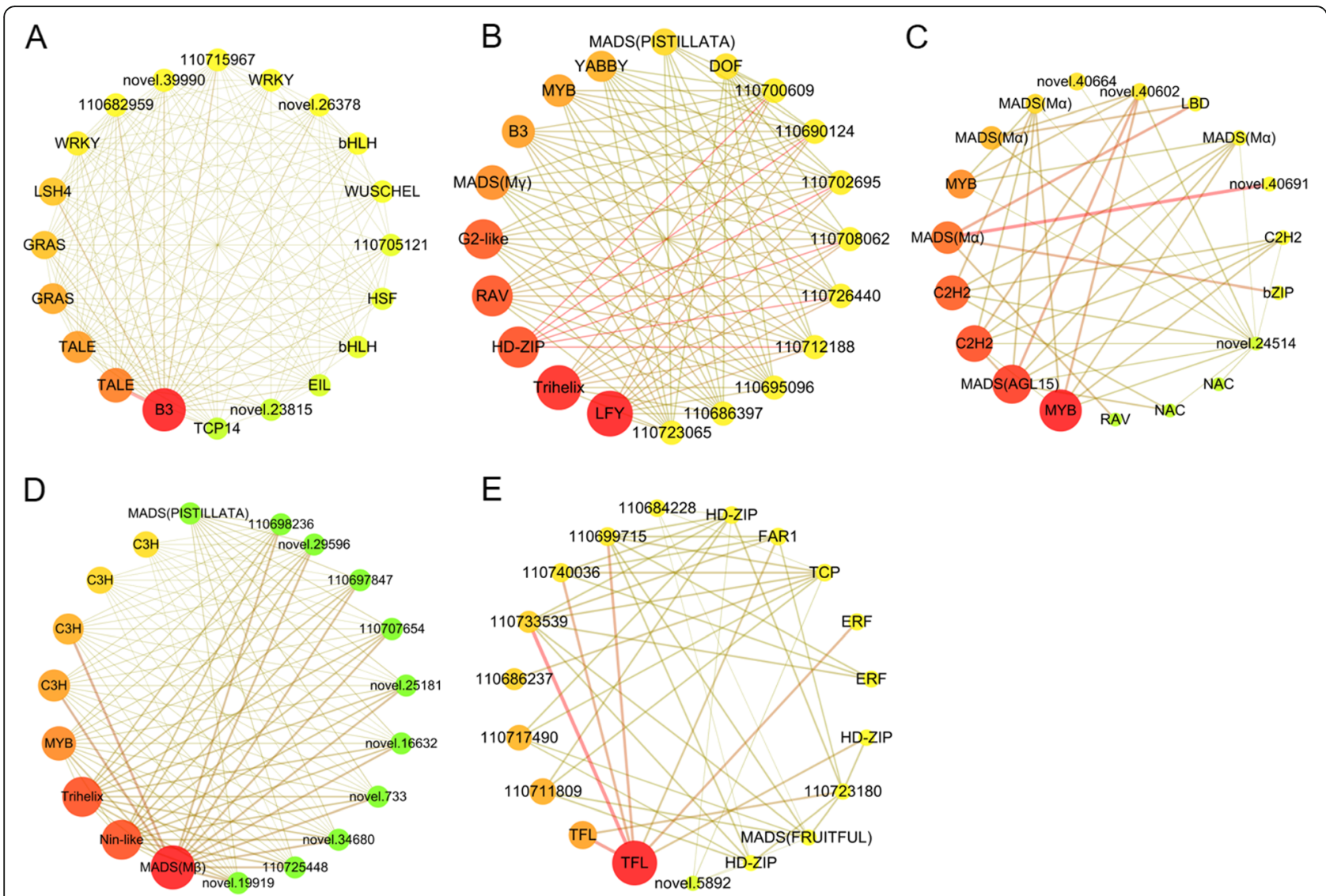

Fig. 7 Central hubs of the core co-expression networks involved in maintaining young-status and mature-status inflorescence. The core regulatory network was generated from each module with stringent edge weight cutoffs by using the TFs and crucial plant inflorescence development related homologs as seed nodes. The nodes with top degrees were defined as central hubs in each core network. a for the Green module, a core network composed of 602 nodes and 2253 edges were generated using an edge weight cutoff of 0.50; (b) for the Pink module, a core network composed of 111 nodes and 394 edges was generated using an edge weight cutoff of 0.30; (c) for the Blue module, a core network composed of 649 nodes and 2055 edges was generated using an edge weight cutoff of 0.58; (d) for the Black module, a core network composed of 227 nodes and 1021 edges was generated using an edge weight cutoff of 0.55; (e) for the Turquoise module, a core network composed of 232 nodes and 499 edges was generated using an edge weight cutoff of 0.58 . The networks were visualized using Cytoscape. The nodes with larger size and darker red indicate greater connectivity within the network

effective analysis methods. WGCNA is a very powerful tool for identifying key regulators in big data analysis. Recently, its application has elucidated the complex networks of many important agronomic traits, such as wheat spike architecture [59], wild strawberry flower and fruit development [60], and chickpea seed development [61].

In this study, by using WGCNA, 21,610 DEGs identified across all developmental stages were divided into 10 modules. According to the expression profiles of these modules, we found gene sets in five modules representing early (YP1) and late induction (P1/P2) of gene expression. Expression levels of the Green and Pink modules peaked at young panicles (YP1), and the Turquoise, Blue, Black and Purple modules showed specific expression at late reproductive phase panicles (P1 and P2). As YP1 inflorescences showed no obvious branching phenotypes compared with P1 and P2 inflorescences, we designated that YP1 inflorescences were not branched whereas P1 and P2 inflorescences had experienced branching. Consequently, we mainly explored YP1, P1 and P2 associated modules. KEGG enrichment analysis of the early induced modules (Green/Pink) highlights the roles of "Plant hormone signal transduction", "Ribosome" and "Photosynthesis" at YP1 stage, while the late induced module (Turquoise) is highly enriched in "Carbon fixation in photosynthetic organisms" and "Carbon metabolism", suggesting phytohormone signaling is essential for inflorescence development before branching while carbon source energy is important for branching occurrence.

Transcriptional regulation has pivotal role in inflorescence development, up to date most of the unveiled inflorescence architecture regulators are TF encoding genes [4]. Therefore, in the present study we emphasized on investigating the TF associated subnetworks. Before 


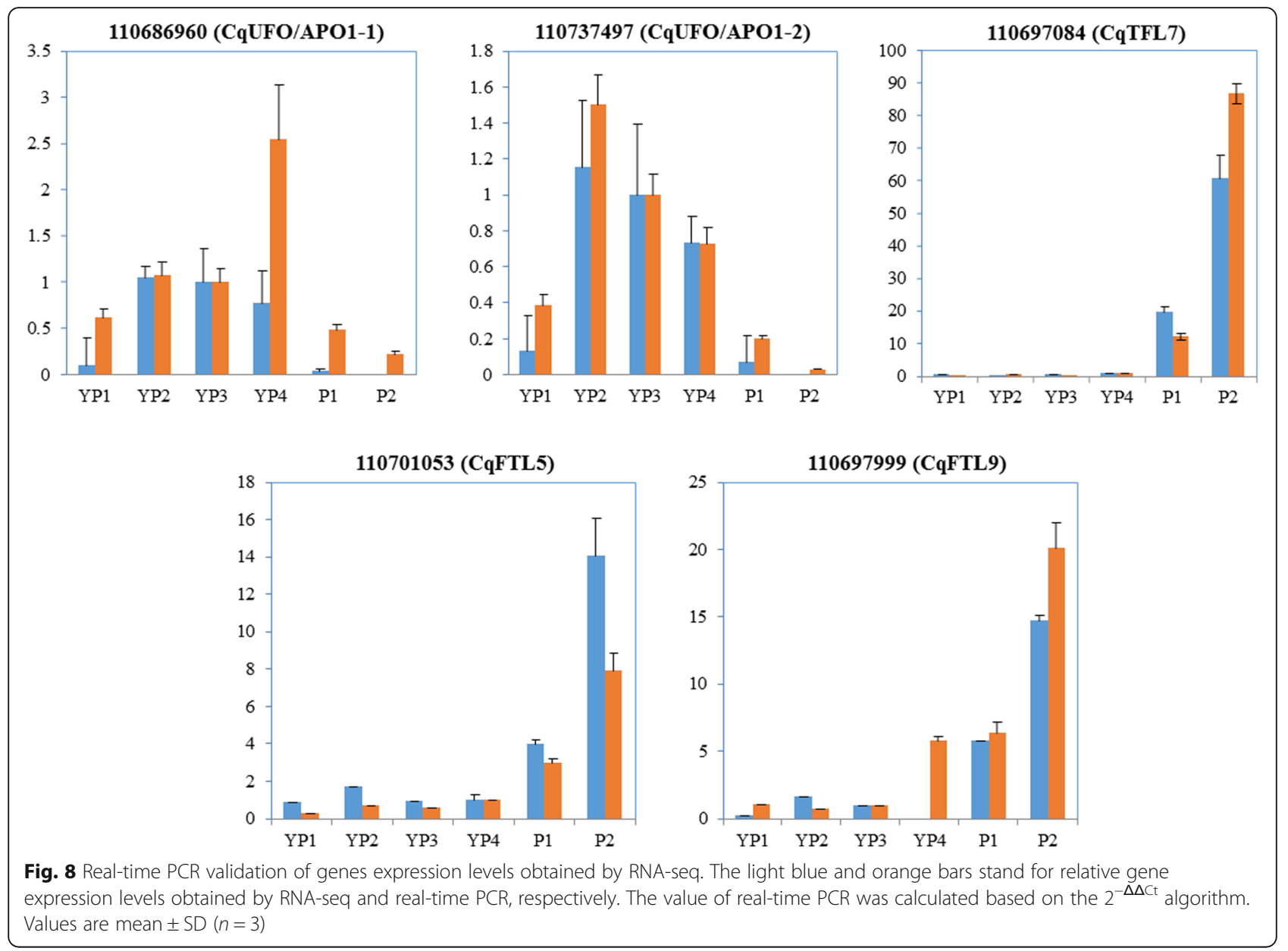

construction of core networks, we manually refined the networks with stringent cutoffs to filter out edges with low edge weights. Then, we used the TFs and the key inflorescence-shaping related homologs as seed nodes to extract the coexpression networks. As hub genes are principle regulators in modules, we designated the 20 genes with top connectivity as central hubs in each network. Clearly, most hub components in early reproductive phase related modules are different from that in late reproductive phase related modules. For example, B3, TALE, WOX, LSH, LFY, GRAS, bHLH, EIL, DOF, G2-like and $Y A B B Y$ family members uniquely occurred in the Green and Pink modules, whereas TFL, ERF, bZIP, HDZIP, C2H2, LBD, NAC, C3H, Nin-like and FAR1 specifically occurred in the Blue, Black and Turquoise modules as central hubs. Consistently, some of these hub homologs were deciphered as inflorescence controllers in previous cases. Mutation of the TALE family member KNOX and the WOX family member WUS renders smaller inflorescence and less branching morphologies $[15,18,62]$. Gain-of-function mutation in the $L S H$ family member TAW1 causes prolonged branching period and increased yield [35]. Overexpression of a rice
DOF member-OsDOF12 leads to reduced panicle size [63]. The GRAS family member MOC1 is required for tiller and panicle branching in rice [54]. $L F Y$ functions as floral identity gene in Arabidopsis and its homolog $A P O 2 / R F L$ functions as flowering time controller and panicle architecture modulator in rice [34]. TFL antagonizes with $A P 1$ to control floral transition and inflorescence architecture [30]. The ERF family member FZP/ BFL1 inhibits axillary meristems and activates florets specification [37]. Maize RAMOSA 1 (RA1, [64]) and $R A 2$ [65] are involved in inflorescence development, belonging to $C 2 H 2$ and $L B D$ family, respectively. Several cases indicate that $\mathrm{C} 2 \mathrm{H} 2$ and $\mathrm{C} 3 \mathrm{H}$ genes not only participate in controlling flowering time, but also are involved in regulating panicle structure in rice $[66,67]$. Thus, we speculate that the unique distribution pattern of these TF nodes may account for the distinct developmental status of non-branching (YP1) and branched (P1 and P2) inflorescence. MADS genes have versatile roles in regulating flowering time, inflorescence architecture, floral specification, and fruit and seed development $[58,68]$. In this case, we found MADS genes occurred in both none-branching and branched status 
samples, and the MADS nodes in branched inflorescences outnumbered that associated with none-branching inflorescences. Coincidently, recent transcriptome profiling studies also identified several $M A D S$ genes associated with spike architecture in wheat and barley (Hordeum vulgare) [69]. In our study, the identified $M A D S$ genes are inclusive of $M \alpha, M \beta, M \gamma$, PISTILLATA-like, FRUITFULL-like and AGL18-like subfamilies. The detailed information regarding to the genetic roles of these $M A D S$ family members has been well reviewed [58,68], indicating overlapping and divergent roles exist between different subfamilies. Therefore, we postulate that the different functions of these $M A D S$ members may be responsible for the inflorescence development and branching. Further functional characterization of these hub genes in the future will shed light on how they interact with each other to modulate quinoa inflorescence architecture.

\section{Conclusions}

In the present study we carried out transcriptome sequencing of six different stages inflorescence samples. We emphasized on analyzing the modules highly associated with early and late reproductive stages to generate core coexpression networks underlying the inflorescence architecture using weighted gene co-expression network analysis (WGCNA). Based on the WGCNA results, we speculate that the B3 (110723560) in the Green module and the LFY (110698679) in the Pink module are the most important regulators for the induction and maintenance of non-branching inflorescence, whereas the $M Y B$ (novel.11405) in the Blue module, the M-type MADS (novel.4708) in the Black module and the TFL (110697084) in the Turquoise module are essential for the inflorescence branching at late reproductive phase. Overall, the data obtained from this study will significantly contribute to our understanding of the networks regulating quinoa inflorescence architecture, as well will supply with valuable genetic resources for molecular breeding in the future.

\section{Methods}

\section{Plant materials and growth conditions}

Quinoa (Chenopodium quinoa Willd.) cultivator "LL-1" was used in this study. LL-1 was derived from a Bolivia quinoa cultivar "Puno" and was self-pollinated for at least six generations in the Experimental Station of Key Laboratory of Coarse Cereal Processing, Ministry of Agriculture in Jintang, Chengdu. The quinoa seeds were sown in a pot with $20 \mathrm{~cm}(\mathrm{~cm})$ in diameter and $18 \mathrm{~cm}$ in depth. The sowing dates were July 19th, July 29th, August 24th, September 2nd, September 9th and September 16th in 2017. Seven days after sowing, the most robust five shoots in each pot were retained by removing the spared ones. All the quinoa plants were maintained in a greenhouse at Chengdu University $\mathrm{N} 30^{\circ} 39^{\prime}$, E104 $11^{\prime}$ ) with regular management, supplied with $200 \mathrm{mg} / \mathrm{L}$ water-soluble compound fertilizer $\left(\mathrm{N}: \mathrm{P}_{2} \mathrm{O}_{5}: \mathrm{K}_{2} \mathrm{O}=16: 7: 22\right)$ every 3 weeks and watered every 2 days. The quinoa plants were grown under natural photoperiod conditions which were approximately 13.8 $\mathrm{h}, 13.2 \mathrm{~h}, 12.3 \mathrm{~h}, 11.4 \mathrm{~h}, 10.6 \mathrm{~h}$ and $10.1 \mathrm{~h}$ in the middle of July, August, September, October, November and December in 2017, respectively. We manually removed the bracts using scalpel once a week to observe whether the plants had born floral buds. The young inflorescences (sown on August 24th, September 2nd, September 9th and September 16th in 2017, respectively referred to as YP4, YP3, YP2 and YP1) of five plants and the late-stage inflorescences (sowed on July 19th and July 29th, respectively referred to as P2 and P1) of three plants were collected on December 15th, three biological replicates for each occasion. The harvested samples were immediately stored in liquid nitrogen before total RNA extraction.

\section{RNA extraction and library preparation for high throughput sequencing}

Total RNA of the samples collected above was extracted by using TRIzol reagent (Invitrogen) according to the manufacture's instruction. The RNA concentration, purity and integrity were measured using Qubit RNA Assay Kit in Qubit $^{\circ} 2.0$ Flurometer (Life Technologies, CA, USA), NanoPhotometer ${ }^{\circ}$ spectrophotometer (IMPLEN, CA, USA) and the RNA Nano 6000 Assay Kit of the Bioanalyzer 2100 system (Agilent Technologies, CA, USA), respectively. The RNA samples whose RIN (RNA Integrity Number) values larger than 8.5 were used for following sequencing library construction. By applying poly-T oligo-attached magnetic beads, the mRNA from $3 \mu \mathrm{g}$ total RNA were isolated and purified. After fragmentation and double-strand cDNA synthesis, sequencing libraries were constructed using NEBNext ${ }^{\circ}$ UltraTM RNA Library Prep Kit for Illumina (NEB, USA) following the manufacturer's protocols.

\section{RNA sequencing and data analysis}

RNA sequencing was performed on an Illumina platform, and approximately 22 million to 31 million of paired-end $150 \mathrm{bp}$ raw reads were generated. After removing the adaptor, low-quality and poly- $\mathrm{N}$ containing reads from raw reads, clean reads were obtained and used for downstream data analysis. Meanwhile, Q20, Q30 and GC content of the clean reads for each sample were calculated. Using HISAT (v2.0.5) [47] with default parameters, paired-end clean reads were mapped to the Chenopodium quinoa reference genome sequences [42] (http:/ftp.ncbi.nlm.nih.gov/genomes/all/GCF/001/683/475/ GCF_001683475.1_ASM168347v1). At the same time, StringTie (v1.3.3b) [48] was adopted to predict novel 
transcripts based on a novel network flow algorithm as well as an optional de novo assembly step. Subsequently, the novel genes were annotated through Pfam database [70]. For gene expression level analysis, Feature Counts (v1.5.0p3) [71] was applied to count the reads numbers mapped to each gene, and FPKM (Fragments Per Kilobase of transcript sequence per Millions base pairs sequenced) value of each gene was calculated [49]. The Pearson correlations between biological replicates were calculated based on the FPKM values using the $\mathrm{R}$ function cor, and the result showed that all gene expression levels between replicates were highly correlated (R2 $>0.916)$. DESeq2 R package (1.16.1) [50] was used to normalize the read count value and dig out the differentially expressed genes (DEGs) between the groups (each with three biological replicates) at different developmental stages. The resulting $P$ values were adjusted using the Benjamini and Hochberg's approach for controlling the false discovery rate. An adjusted $P$-value less than 0.05 was applied to call DEGs between different groups.

\section{GO and KEGG enrichment analysis of DEGs}

To understand the potential biological functions of DEGs, we used cluster Profiler R package to carry out Gene Ontology (GO) [72] and Kyoto Encyclopedia of Genes and Genomes (KEGG) [73] enrichment analysis. GO or KEGG terms with corrected $P$-value less than 0.05 were considered significantly enriched by DEGs.

\section{Transcription factor prediction of DEGs}

To identify transcription factor encoding genes among DEGs, we adopted the Transcription Factor Prediction tool in Plant Transcription Factor Database v4.0 [51] (http://planttfdb.cbi.pku.edu.cn). ESTScan [74] was employed to identify and translate the Coding Sequence regions of the uploaded nucleic acid sequences. The defaulted parameters for TF family assignment and thresholds were adopted to identify transcrption factors from the DEG sequences.

\section{Construction of core regulatory network}

All the DEGs were used to construct gene co-expression networks based on the expression levels (FPKM) by applying the Weighted Gene Co-Expression Network Analysis (WGCNA) [52] package in R. With default parameters (unsigned-type topological overlap matrix (TOM), power $\beta$ was set to 6 , minimal module size was set to 30 , merge cut height was set to 0.25 ), the automatic network construction and module detection method were used for gene co-expression network construction. The edge weight (ranging from 0 to 1 ) which indicates the strength of the connectivity between two genes was calculated. Then, 21,568 out of 21,610 DEGs were clustered in 10 different modules. For core network construction, edge weight thresholds of $0.5,0.3,0.58$, 0.55 , and 0.58 were applied for refining the Green, Pink, Blue, Black and Turquoise module (Additional file 12: Dataset 1). Subsequently, TF encoding genes and key inflorescence architecture regulators in each module were used as seed nodes to generate core transcriptional regulatory network. Then, the core networks were visualized and analyzed using Cytoscape [53], and the genes with top connective degrees were defined as central hubs.

\section{Real-time PCR}

The total RNA of each sample was extracted using TRIzol reagent. Two micrograms of total RNA for each sample were used for DNA digestion and reverse transcription to synthesize first-strand cDNA using EasyScript One-Step gDNA Removal and cDNA Synthesis SuperMix Kit (Transgen Biotech, Beijing, China). Subsequently, the properly diluted cDNA was used to perform real-time PCR by applying TransStart Green qPCR SuperMix (Transgen Biotech) on a real-time PCR system (LineGene 9600, BIOER, Hangzhou, China). The real-time PCR program was: $94^{\circ} \mathrm{C}$ for $5 \mathrm{~min}, 39$ cycles of denaturing at $95^{\circ} \mathrm{C}$ for $5 \mathrm{~s}$, annealing and extension at $59^{\circ} \mathrm{C}$ for $20 \mathrm{~s}$. A quinoa $M O N$ gene [75] (Monensin Sensitivity 1, Gene ID: 110720838) was used as the reference gene. The relative expression level of detected genes was quantified by the $2^{-\Delta \Delta \mathrm{Ct}}$ algorithm from three replicates using Microsoft Office Excel 2016 (Washington, USA). The related primers used for real-time PCR were list in Additional file 11: Table S9. Then, the two-scale expression values (RNA-seq (FPKM) and real-time PCR) was normalized to be identical. For CqUFO/APO1-1, APO12, CqTFL7 and CqFTL9, the gene expression values in YP3 obtained by real-time PCR and RNA-seq were both normalized to 1 , and then the expression levels in other points (YP1, YP2, YP4, P1 and P2) were calculated according to the fold changes. For CqFTL5, the expression levels were calculated with the expression value in YP4 normalized to 1 .

\section{Additional files}

Additional file 1: Figure S1. Correlation coefficient between three biological replicates or between samples collected at different stages. The value was calculated based on the gene expression profiles using the $R$ function cor. (DOCX $2439 \mathrm{~kb}$ )

Additional file 2: Figure S2. The TF percentages in DEGs and in quinoa genome. The TFs were identified using the Transcription Factor Prediction tool in Plant Transcription Factor Database v4.0, and then the proportion of TFs in DEGs (A) and the TF family percentages in quinoa genome (B) was calculated. (DOCX $1822 \mathrm{~kb}$ )

Additional file 3: Table S1. Statistic of the expressed genes at each stage. (DOCX $19 \mathrm{~kb}$ )

Additional file 4: Table S2. Statistic of the DEGs number between different samples. (DOCX $19 \mathrm{~kb}$ ) 
Additional file 5: Table S3. GO enrichment analysis of the DEGs at different intervals. (XLSX $50 \mathrm{~kb}$ )

Additional file 6: Table S4. Transcription factors identified in DEGs at different intervals. (XLSX $16 \mathrm{~kb}$ )

Additional file 7: Table S5. List of Transcription factors in quinoa genome. (XLSX $153 \mathrm{~kb}$ )

Additional file 8: Table S6. Genes distributed in different modules. (XLSX $5751 \mathrm{~kb}$ )

Additional file 9: Table S7. Transcription factors identified in different modules. (XLSX $33 \mathrm{~kb}$ )

Additional file 10: Table S8. The key genes used as seed nodes to generate core transcriptional regulatory network. (XLSX $16 \mathrm{~kb}$ )

Additional file 11: Table S9. The primer sequences used for real-time $P C R$ in this study. (DOCX $17 \mathrm{~kb}$ )

Additional file 12: Dataset 1. The sequences of novel genes identified in this study. (TXT $41244 \mathrm{~kb}$ )

Additional file 13: Dataset 2. List of nodes included in different modules with stringent edge weight thresholds used for construction of gene regulatory networks. (XLSX $2433 \mathrm{~kb}$ )

\section{Abbreviations}

AGL24: AGAMOUS-LIKE 24; ALOG: Arabidopsis LSH1 and Oryza G1; AMs: Axillary meristems; AP1: APETALA 1; APO1: ABERRANT PANICLE ORGANIZATION 1; BD1: BRANCHED SILKLESS1; BFL1: BRANCHED FLORETLESS 1; BMs: Branch meristems; BRC1: BRANCHED1; CLE: CLV3/ESR-related; CLV: CLAVATA; CO: CONSTANS; DAS: Days after sowing; DEGs: Differentially expressed genes; ERF: Ethylene-response factor; FEA2: FASCIATED EAR 2; FM: Floral meristems; FON1: FLORAL ORGAN NUMBER 1; FPKM: Fragments Per Kilobase of transcript sequence per Millions base pairs sequenced; FZP: FRIZZY PANICLE; GO: Gene ontology; IDS1: INDETERMINATE SPIKELET 1 ;

IM: Inflorescence meristem; IPA1: Ideal Plant Architecture 1; KEGG: Kyoto Encyclopedia of Genes and Genomes; KN1: KNOTTED 1; KNOX: KNOTTED 1-like homeobox; LFY: LEAFY; MFS1: MULTI-FLORET SPIKELET 1; MOC1: MONOCULM 1 MOS1: MORE SPIKELETS 1; OSH1: Oryza sativa HOMEOBOX 1; PAP2: PANICLE PHYTOMER 2; PEBP: Phosphatidylethanolamine-binding protein;

QTL: Quantitative trait loci; RA1: RAMOSA1; RCN1: REDUCED CULM NUMBER 1; RFL: RICE FLORICAULA; SAM: Shoot apical meristem; SBP: SQUAMOSA promoter binding protein-like; SEP: SEPALLATA; SNB: SUPERNUMERARY BRACT; SOC1: SUPPRESSOR OF OVEREXPRESSION OF CONSTANS 1; STM: SHOOT MERISTEMLESS; SVP: SHORT VEGETATIVE PHASE; TCP: TEOSINTE BRANCHED, CYCLOIDEA and PCF; TD1: THICK TASSEL DWARF 1; TF: Transcription factor; TFL1: TERMINAL FLOWER 1; TMF: TERMINATING FLOWER; TSH4: TASSELSHEATH4; TWA1: TAWAWA1; UFO: UNUSUAL FLORAL ORGAN; WFP: Wealthy Farmer's Panicle; WGCNA: Weighted Gene Co-Expression Network Analysis; WOX: WUSCHEL-related homeobox; WUS: WUSCHEL; ZCN1: CENTRORADIALIS 1

\section{Acknowledgements}

We thank Dr. Guixing Ren (Institute of Crop Sciences, Chinese Academy of Agricultural Sciences) for providing us with quinoa seeds of "LL-1".

\section{Authors' contributions}

QW conceived and designed this study. QW wrote the manuscript. QW, XB and WZ performed most of the experiments. XS, XW and YS participated in analyzing the RNA-seq data. DX and YW managed the quinoa plants and collected the samples for sequencing. JZ, LP and GZ extracted the total RNA and performed real-time PCR. All authors have read and approved the final manuscript.

\section{Funding}

This work is supported by the National Natural Science Foundation of China (Grant 31701493); the Sichuan Science and Technology Program (Grant 2018JY0344); the Sichuan Education Department Program (Grant 18ZA0132); the China Agriculture Research System (Project CARS-08-02A) and Chengdu University Research Fund (Grant 2017XJZ19). The funding bodies have no role in the design of the study and collection, analysis, and interpretation of data and in writing the manuscript.

\section{Availability of data and materials}

The RNA-seq data generated from Chenododium quinoa inflorescence samples in this study are deposited at NCBI SRA database (http://trace.ncbi.nlm nih.gov/Traces/sra) under accessions SRR8361800, SRR8361801, SRR8361802, SRR8361803, SRR8361804 and SRR8361805.

Ethics approval and consent to participate

Not applicable.

\section{Consent for publication}

Not applicable.

\section{Competing interests}

The authors declare that they have no competing interests.

Received: 19 December 2018 Accepted: 12 August 2019

Published online: 17 August 2019

\section{References}

1. Xing Y, Zhang Q. Genetic and molecular bases of rice yield. Annu Rev Plant Biol. 2010;61:421-42

2. Wang Y, Li J. Molecular basis of plant architecture. Annu Rev Plant Biol. 2008;59:253-79.

3. Teo ZW, Song S, Wang YQ, Liu J, Yu H. New insights into the regulation of inflorescence architecture. Trends Plant Sci. 2014;19(3):158-65.

4. Zhang D, Yuan Z. Molecular control of grass inflorescence development. Annu Rev Plant Biol. 2014;65:553-78.

5. Wang B, Smith SM, Li J. Genetic regulation of shoot architecture. Annu Rev Plant Biol. 2018.

6. Pautler M, Tanaka W, Hirano HY, Jackson D. Grass meristems I: shoot apical meristem maintenance, axillary meristem determinacy and the flora transition. Plant Cell Physiol. 2013;54(3):302-12

7. Tanaka W, Pautler M, Jackson D, Hirano HY. Grass meristems II: inflorescence architecture, flower development and meristem fate. Plant Cell Physiol. 2013:54(3):313-24

8. Clark SE, Williams RW, Meyerowitz EM. The CLAVATA1 gene encodes a putative receptor kinase that controls shoot and floral meristem size in Arabidopsis. Cell. 1997:89(4):575-85

9. Fletcher JC, Brand U, Running MP, Simon R, Meyerowitz EM. Signaling of cell fate decisions by CLAVATA3 in Arabidopsis shoot meristems. Science. 1999;283(5409):1911-4

10. Jeong S, Trotochaud AE, Clark SE. The Arabidopsis CLAVATA2 gene encodes a receptor-like protein required for the stability of the CLAVATA1 receptorlike kinase. Plant Cell. 1999:11(10):1925-34.

11. Suzaki T, Sato M, Ashikari M, Miyoshi M, Nagato Y, Hirano HY. The gene FLORAL ORGAN NUMBER1 regulates floral meristem size in rice and encodes a leucine-rich repeat receptor kinase orthologous to Arabidopsis CLAVATA1. Development. 2004;131(22):5649-57.

12. Chu H, Qian Q, Liang W, Yin C, Tan H, Yao X, Yuan Z, Yang J, Huang H, Luo $D$, et al. The floral organ number4 gene encoding a putative ortholog of Arabidopsis CLAVATA3 regulates apical meristem size in rice. Plant Physiol. 2006;142(3):1039-52.

13. Bommert $P$, Lunde $C$, Nardmann J, Vollbrecht $E$, Running $M_{1}$ Jackson $D$, Hake S, Werr W. Thick tassel dwarf1 encodes a putative maize ortholog of the Arabidopsis CLAVATA1 leucine-rich repeat receptor-like kinase. Development. 2005;132(6):1235-45.

14. Taguchi-Shiobara F, Yuan Z, Hake S, Jackson D. The fasciated ear2 gene encodes a leucine-rich repeat receptor-like protein that regulates shoot meristem proliferation in maize. Genes Dev. 2001:15(20):2755-66.

15. Schoof $H$, Lenhard M, Haecker A, Mayer KF, Jurgens G, Laux T. The stem cell population of Arabidopsis shoot meristems in maintained by a regulatory loop between the CLAVATA and WUSCHEL genes. Cell. 2000;100(6):635-44.

16. Somssich $M$, Je BI, Simon R, Jackson D. CLAVATA-WUSCHEL signaling in the shoot meristem. Development. 2016:143(18):3238-48.

17. Barazesh S, McSteen P. Hormonal control of grass inflorescence development. Trends Plant Sci. 2008;13(12):656-62.

18. Tsuda K, Ito Y, Sato Y, Kurata N. Positive autoregulation of a KNOX gene is essential for shoot apical meristem maintenance in rice. Plant Cell. 2011; 23(12):4368-81. 
19. Kerstetter RA, Laudencia-Chingcuanco D, Smith LG, Hake S. Loss-of-function mutations in the maize homeobox gene, knotted1, are defective in shoot meristem maintenance. Development. 1997;124(16):3045-54.

20. Long JA, Moan El, Medford JI, Barton MK. A member of the KNOTTED class of homeodomain proteins encoded by the STM gene of Arabidopsis. Nature. 1996:379(6560):66-9.

21. Gao X, Liang W, Yin C, Ji S, Wang H, Su X, Guo C, Kong H, Xue H, Zhang D. The SEPALLATA-like gene OSMADS34 is required for rice inflorescence and spikelet development. Plant Physiol. 2010;153(2):728-40.

22. Kobayashi K, Yasuno N, Sato Y, Yoda M, Yamazaki R, Kimizu M, Yoshida H, Nagamura $Y$, Kyozuka J. Inflorescence meristem identity in rice is specified by overlapping functions of three AP1/FUL-like MADS box genes and PAP2, a SEPALLATA MADS box gene. Plant Cell. 2012;24(5):1848-59.

23. Liljegren SJ, Gustafson-Brown C, Pinyopich A, Ditta GS, Yanofsky MF. Interactions among APETALA1, LEAFY, and TERMINAL FLOWER1 specify meristem fate. Plant Cell. 1999;11(6):1007-18.

24. Wagner D, Sablowski RW, Meyerowitz EM. Transcriptional activation of APETALA1 by LEAFY. Science. 1999;285(5427):582-4.

25. Monniaux M, McKim SM, Cartolano M, Thevenon E, Parcy F, Tsiantis M, Hay A. Conservation vs divergence in LEAFY and APETALA1 functions between Arabidopsis thaliana and Cardamine hirsuta. New Phytol. 2017;216(2):549-61.

26. Nakagawa M, Shimamoto K, Kyozuka J. Overexpression of RCN1 and RCN2, rice TERMINAL FLOWER 1/CENTRORADIALIS homologs, confers delay of phase transition and altered panicle morphology in rice. Plant J. 2002;29(6): 743-50.

27. Danilevskaya ON, Meng X, Ananiev EV. Concerted modification of flowering time and inflorescence architecture by ectopic expression of TFL1-like genes in maize. Plant Physiol. 2010;153(1):238-51.

28. Alvarez J, Guli CL, Yu X-H, Smyth DR. Terminal flower: a gene affecting inflorescence development in Arabidopsis thaliana. Plant J. 1992;2(1):103-16.

29. Souer E, van der Krol A, Kloos D, Spelt C, Bliek M, Mol J, Koes R. Genetic control of branching pattern and floral identity during Petunia inflorescence development. Development. 1998;125(4):733-42.

30. Liu C, Teo ZW, Bi Y, Song S, Xi W, Yang X, Yin Z, Yu H. A conserved genetic pathway determines inflorescence architecture in Arabidopsis and rice. Dev Cell. 2013;24(6):612-22.

31. Jiao Y, Wang Y, Xue D, Wang J, Yan M, Liu G, Dong G, Zeng D, Lu Z, Zhu X, et al. Regulation of OsSPL14 by OsmiR156 defines ideal plant architecture in rice. Nat Genet. 2010;42(6):541-4.

32. Chuck G, Whipple C, Jackson D, Hake S. The maize SBP-box transcription factor encoded by tasselsheath4 regulates bract development and the establishment of meristem boundaries. Development. 2010;137(8):1243-50.

33. Ikeda K, Nagasawa N, Nagato Y. ABERRANT PANICLE ORGANIZATION 1 temporally regulates meristem identity in rice. Dev Biol. 2005;282(2):349-60.

34. Ikeda-Kawakatsu K, Maekawa M, Izawa T, Itoh J, Nagato Y. ABERRANT PANICLE ORGANIZATION 2/RFL, the rice ortholog of Arabidopsis LEAFY, suppresses the transition from inflorescence meristem to floral meristem through interaction with APO1. Plant J. 2012;69(1):168-80.

35. Yoshida A, Sasao M, Yasuno N, Takagi K, Daimon Y, Chen R, Yamazaki R, Tokunaga $H$, Kitaguchi $Y$, Sato $Y$, et al. TAWAWA1, a regulator of rice inflorescence architecture, functions through the suppression of meristem phase transition. Proc Natl Acad Sci U S A. 2013;110(2):767-72.

36. MacAlister CA, Park SJ, Jiang K, Marcel F, Bendahmane A, Izkovich Y, Eshed $Y$, Lippman ZB. Synchronization of the flowering transition by the tomato TERMINATING FLOWER gene. Nat Genet. 2012;44(12):1393-8.

37. Zhu QH, Hoque MS, Dennis ES, Upadhyaya NM. Ds tagging of BRANCHED FLORETLESS 1 (BFL1) that mediates the transition from spikelet to floret meristem in rice (Oryza sativa L). BMC Plant Biol. 2003;3:6.

38. Lee DY, An G. Two AP2 family genes, supernumerary bract (SNB) and Osindeterminate spikelet 1 (OsIDS1), synergistically control inflorescence architecture and floral meristem establishment in rice. Plant J. 2012;69(3): 445-61.

39. Ren D, Li Y, Zhao F, Sang X, Shi J, Wang N, Guo S, Ling Y, Zhang C, Yang Z, et al. MULTI-FLORET SPIKELET1, which encodes an AP2/ERF protein, determines SPIKELET meristem fate and sterile lemma identity in rice. Plant Physiol. 2013;162(2):872-84

40. Chuck G, Meeley R, Hake S. Floral meristem initiation and meristem cell fate are regulated by the maize AP2 genes ids1 and sid1. Development. 2008; 135(18):3013-9.

41. Derbyshire $P$, Byrne ME. MORE SPIKELETS1 is required for spikelet fate in the inflorescence of Brachypodium. Plant Physiol. 2013;161(3):1291-302.
42. Jarvis DE, Ho YS, Lightfoot DJ, Schmockel SM, Li B, Borm TJ, Ohyanagi H, Mineta K, Michell CT, Saber N, et al. The genome of Chenopodium quinoa. Nature. 2017:542(7641):307-12.

43. Wu G. Nutritional Properties of Quinoa. In: Quinoa: Improvement and Sustainable Production. Hoboken: John Wiley \& Sons, Inc; 2015. p. 193-210.

44. Murphy KM, Bazile D, Kellogg J, Rahmanian M. Development of a worldwide consortium on evolutionary participatory breeding in quinoa. Front Plant Sci. 2016;7:608.

45. Bazile D, Jacobsen SE, Verniau A. The global expansion of quinoa: trends and limits. Front Plant Sci. 2016;7:622.

46. Bertero D, Medan D, Hall AJ. Changes in Apical Morphology during Floral Initiation and Reproductive Development in Quinoa (Chenopodium quinoa Willd.). Ann Bot. 1996:78(3):317-24.

47. Kim D, Langmead B, Salzberg SL. HISAT: a fast spliced aligner with low memory requirements. Nat Methods. 2015;12(4):357-60.

48. Pertea M, Pertea GM, Antonescu CM, Chang TC, Mendell JT, Salzberg SL. StringTie enables improved reconstruction of a transcriptome from RNA-seq reads. Nat Biotechnol. 2015;33(3):290-5

49. Trapnell C, Williams BA, Pertea G, Mortazavi A, Kwan G, van Baren MJ, Salzberg SL, Wold BJ, Pachter L. Transcript assembly and quantification by RNA-Seq reveals unannotated transcripts and isoform switching during cell differentiation. Nat Biotechnol. 2010;28(5):511-5.

50. Love Ml, Huber W, Anders S. Moderated estimation of fold change and dispersion for RNA-seq data with DESeq2. Genome Biol. 2014;15(12):550.

51. Jin J, Tian F, Yang DC, Meng YQ, Kong L, Luo J, Gao G. PlantTFDB 4.0: toward a central hub for transcription factors and regulatory interactions in plants. Nucleic Acids Res. 2017;45(D1):D1040-5.

52. Langfelder P, Horvath S. WGCNA: an R package for weighted correlation network analysis. BMC Bioinformatics. 2008;9:559.

53. Shannon P, Markiel A, Ozier O, Baliga NS, Wang JT, Ramage D, Amin N, Schwikowski B, Ideker T. Cytoscape: a software environment for integrated models of biomolecular interaction networks. Genome Res. 2003;13(11): 2498-504

54. Li X, Qian Q, Fu Z, Wang Y, Xiong G, Zeng D, Wang X, Liu X, Teng S, Hiroshi $F$, et al. Control of tillering in rice. Nature. 2003;422(6932):618-21.

55. Hubbard L, McSteen P, Doebley J, Hake S. Expression patterns and mutant phenotype of teosinte branched1 correlate with growth suppression in maize and teosinte. Genetics. 2002;162(4):1927-35.

56. Aguilar-Martinez JA, Poza-Carrion C, Cubas P. Arabidopsis BRANCHED1 acts as an integrator of branching signals within axillary buds. Plant Cell. 2007; 19(2):458-72

57. Benlloch R, Berbel A, Ali L, Gohari G, Millan T, Madueno F. Genetic control of inflorescence architecture in legumes. Front Plant Sci. 2015;6:543.

58. Smaczniak C, Immink RG, Angenent GC, Kaufmann K. Developmental and evolutionary diversity of plant MADS-domain factors: insights from recent studies. Development. 2012;139(17):3081-98.

59. Wang $Y$, Yu H, Tian C, Sajjad M, Gao C, Tong Y, Wang X, Jiao Y. Transcriptome association identifies regulators of wheat spike architecture. Plant Physiol. 2017:175(2):746-57.

60. Shahan R, Zawora C, Wight H, Sittmann J, Wang W, Mount SM, Liu Z. Consensus Coexpression network analysis identifies key regulators of flower and fruit development in wild strawberry. Plant Physiol. 2018; 178(1):202-16.

61. Garg R, Singh VK, Rajkumar MS, Kumar V, Jain M. Global transcriptome and coexpression network analyses reveal cultivar-specific molecular signatures associated with seed development and seed size/weight determination in chickpea. Plant J. 2017;91(6):1088-107.

62. Sato Y, Aoki Y, Matsuoka M. A loss-of-function mutation in the rice KNOX type homeobox gene, OSH3. Plant Cell Physiol. 2002;43(1):44-51.

63. Wu Q, Li D, Li D, Liu X, Zhao X, Li X, Li S, Zhu L. Overexpression of OsDof12 affects plant architecture in rice (Oryza sativa L.). Front Plant Sci. 2015;6:833.

64. Gallavotti A, Long JA, Stanfield S, Yang X, Jackson D, Vollbrecht E, Schmidt $\mathrm{RJ}$. The control of axillary meristem fate in the maize ramosa pathway. Development. 2010:137(17):2849-56.

65. Bortiri E, Chuck G, Vollbrecht E, Rocheford T, Martienssen R, Hake S. Ramosa2 encodes a LATERAL ORGAN BOUNDARY domain protein that determines the fate of stem cells in branch meristems of maize. Plant Cell. 2006:18(3):574-85

66. Matsubara K, Yamanouchi U, Wang ZX, Minobe Y, Izawa T, Yano M. Ehd2, a Rice Ortholog of the maize INDETERMINATE1 gene, promotes flowering by up-regulating Ehd1. Plant Physiol. 2008;148(3):1425-35. 
67. Gao H, Zheng XM, Fei G, Chen J, Jin M, Ren Y, Wu W, Zhou K, Sheng P, Zhou F, et al. Ehd4 encodes a novel and oryza-genus-specific regulator of photoperiodic flowering in rice. PLoS Genet. 2013;9(2):e1003281.

68. Schilling S, Pan S, Kennedy A, Melzer R. MADS-box genes and crop domestication: the jack of all traits. J Exp Bot. 2018;69(7):1447-69.

69. Digel B, Pankin A, von Korff M. Global transcriptome profiling of developing leaf and shoot apices reveals distinct genetic and environmental control of floral transition and inflorescence development in barley. Plant Cell. 2015; 27(9):2318-34.

70. Finn RD, Coggill P, Eberhardt RY, Eddy SR, Mistry J, Mitchell AL, Potter SC, Punta M, Qureshi M, Sangrador-Vegas A, et al. The Pfam protein families database: towards a more sustainable future. Nucleic Acids Res. 2016;44(D1): D279-85.

71. Liao Y, Smyth GK, Shi W. FeatureCounts: an efficient general purpose program for assigning sequence reads to genomic features. Bioinformatics. 2014;30(7):923-30.

72. Ashburner M, Ball CA, Blake JA, Botstein D, Butler H, Cherry JM, Davis AP, Dolinski K, Dwight SS, Eppig JT, et al. Gene ontology: tool for the unification of biology. The gene ontology consortium. Nat Genet. 2000;25(1):25-9.

73. Kanehisa M, Goto S. KEGG: Kyoto encyclopedia of genes and genomes. Nucleic Acids Res. 2000;28(1):27-30.

74. Iseli C, Jongeneel CV, Bucher P. ESTScan: a program for detecting, evaluating, and reconstructing potential coding regions in EST sequences. Proc Int Conf Intell Syst Mol Biol. 1999:99:138-48.

75. Fiallos-Jurado J, Pollier J, Moses T, Arendt P, Barriga-Medina N, Morillo E, Arahana V, de Lourdes TM, Goossens A, Leon-Reyes A. Saponin determination, expression analysis and functional characterization of saponin biosynthetic genes in Chenopodium quinoa leaves. Plant Sci. 2016; 250:188-97.

\section{Publisher's Note}

Springer Nature remains neutral with regard to jurisdictional claims in published maps and institutional affiliations.

Ready to submit your research? Choose BMC and benefit from:

- fast, convenient online submission

- thorough peer review by experienced researchers in your field

- rapid publication on acceptance

- support for research data, including large and complex data types

- gold Open Access which fosters wider collaboration and increased citations

- maximum visibility for your research: over $100 \mathrm{M}$ website views per year

At $\mathrm{BMC}$, research is always in progress.

Learn more biomedcentral.com/submissions 